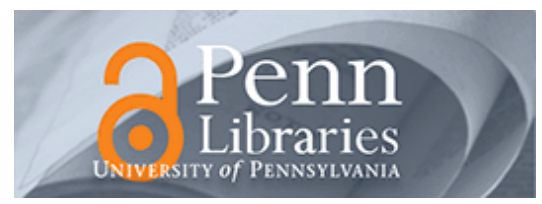

University of Pennsylvania ScholarlyCommons

December 2006

\title{
Linear Time Logic Control of Discrete-Time Linear Systems
}

\author{
Paulo Tabuada \\ University of California \\ George J. Pappas \\ University of Pennsylvania, pappasg@seas.upenn.edu
}

Follow this and additional works at: https://repository.upenn.edu/ese_papers

\section{Recommended Citation \\ Paulo Tabuada and George J. Pappas, "Linear Time Logic Control of Discrete-Time Linear Systems", . December 2006.}

Copyright 2006 IEEE. Reprinted from IEEE Transactions on Automatic Control, Volume 51, Issue 12, pages 1862-1877.

This material is posted here with permission of the IEEE. Such permission of the IEEE does not in any way imply IEEE endorsement of any of the University of Pennsylvania's products or services. Internal or personal use of this material is permitted. However, permission to reprint/republish this material for advertising or promotional purposes or for creating new collective works for resale or redistribution must be obtained from the IEEE by writing to pubs-permissions@ieee.org. By choosing to view this document, you agree to all provisions of the copyright laws protecting it.

This paper is posted at ScholarlyCommons. https://repository.upenn.edu/ese_papers/222

For more information, please contact repository@pobox.upenn.edu. 


\title{
Linear Time Logic Control of Discrete-Time Linear Systems
}

\begin{abstract}
The control of complex systems poses new challenges that fall beyond the traditional methods of control theory. One of these challenges is given by the need to control, coordinate and synchronize the operation of several interacting submodules within a system. The desired objectives are no longer captured by usual control specifications such as stabilization or output regulation. Instead, we consider specifications given by linear temporal logic (LTL) formulas. We show that existence of controllers for discrete-time controllable linear systems and LTL specifications can be decided and that such controllers can be effectively computed. The closed-loop system is of hybrid nature, combining the original continuous dynamics with the automatically synthesized switching logic required to enforce the specification.

\section{Keywords}

automatic synthesis, discrete-time, linear control systems, hybrid systems, linear time logic

Comments

Copyright 2006 IEEE. Reprinted from IEEE Transactions on Automatic Control, Volume 51, Issue 12, pages 1862-1877.

This material is posted here with permission of the IEEE. Such permission of the IEEE does not in any way imply IEEE endorsement of any of the University of Pennsylvania's products or services. Internal or personal use of this material is permitted. However, permission to reprint/republish this material for advertising or promotional purposes or for creating new collective works for resale or redistribution must be obtained from the IEEE by writing to pubs-permissions@ieee.org. By choosing to view this document, you agree to all provisions of the copyright laws protecting it.
\end{abstract}




\title{
Linear Time Logic Control of Discrete-Time Linear Systems
}

\author{
Paulo Tabuada, Member, IEEE, and George J. Pappas, Senior Member, IEEE
}

\begin{abstract}
The control of complex systems poses new challenges that fall beyond the traditional methods of control theory. One of these challenges is given by the need to control, coordinate and synchronize the operation of several interacting submodules within a system. The desired objectives are no longer captured by usual control specifications such as stabilization or output regulation. Instead, we consider specifications given by linear temporal logic (LTL) formulas. We show that existence of controllers for discrete-time controllable linear systems and LTL specifications can be decided and that such controllers can be effectively computed. The closed-loop system is of hybrid nature, combining the original continuous dynamics with the automatically synthesized switching logic required to enforce the specification.
\end{abstract}

Index Terms-Automatic synthesis, discrete-time, linear control systems, hybrid systems, linear time logic.

\section{INTRODUCTION}

\section{A. Motivation}

$\mathbf{I}$ $\mathrm{N}$ RECENT years, there has been an increasing interest in extending the application domain of systems and control theory from monolithic continuous plants to complex systems consisting of several concurrently interacting submodules. Examples range from multimodal software control systems in the aerospace [1], [2] and automotive industry [4], [5] to advanced robotic systems [6], [8]. This change in perspective is accompanied by a shift in control objectives. One is no longer interested in the stabilization or output regulation of individual continuous plants, but rather wishes to regulate the global system behavior through the local control of each individual submodule or component. Typical specifications for this class of control problems include coordination and synchronization of individual modules, sequencing of tasks, reconfigurability and adaptability of components, etc. In order to address this emerging class of control problems we need to formally specify the desired system behavior:

Manuscript received February 9, 2004; revised February 25, 2005, March 1, 2006, and April 12, 2006. Recommended by Associate Editor J. P. Hespanha. The work of P. Tabuada was supported in part by the National Science Foundation under CAREER award 0446716. The work of G. J. Pappas was supported in part by the National Science Foundation under Grant EHS 0311123.

P. Tabuada was with the Department of Electrical Engineering, the University of Notre Dame, Notre Dame, IN 46556 USA. He is now with the Electrical Engineering Department, the University of California, Los Angeles, CA 900951594 (e-mail: tabuada@ee.ucla.edu).

G. J. Pappas is with the Department of Electrical and Systems Engineering, the University of Pennsylvania, Philadelphia, PA 19104 USA (e-mail: pappasg@ee.upenn.edu).

Color version of Fig. 1 available online at http://ieeexplore.ieee.org.

Digital Object Identifier 10.1109/TAC.2006.886494
How can we formally and succinctly specify the desired behavior of concurrently interacting systems?

The specification mechanism should also lead to controller design methods. These controllers will enforce a hybrid behavior on the controlled system since system evolution is influenced both by the continuous dynamics and by the discrete interaction (communication) between submodules. While many ad-hoc approaches have been reported in the literature for the design of such hybrid systems, any formal guarantee of operation can only be obtained through formal verification which is noticeably a hard problem [9]. This suggests that one should aim at design methods that satisfy the specification by construction:

How can we design (hybrid) controllers ensuring satisfaction of specifications by construction, thereby avoiding or substantially reducing the need for formal verification?

Another dimension of this problem, that should not be neglected, is its computational aspect. As the number of modules increases, the possibilities of interaction between modules also increase thus rendering analysis of global behavior an extremely difficult task. This intrinsic complexity of concurrently interacting systems can only be addressed by computational synthesis methods, reducing error-prone human analysis or synthesis to the strictly necessary minimum. Only fully automated methods have the potential to scale and successfully address control problems for systems consisting of large numbers of interacting components:

How can we render the design of controllers completely automated, from specification to implementation?

Motivated by the previously described problems, we present in this paper an approach for the control of linear systems with objectives expressed in linear temporal logic (LTL). There are two main reasons to describe control objectives in temporal logic. Firstly, temporal logic provides a formal specification mechanism allowing one to quantitatively define the desired behavior of a systems by prescribing the interaction between submodules. Secondly, temporal logic makes it possible to succinctly express complex objectives due to its similarity to natural language. In particular, temporal logic is well suited to express the novel class of specifications required by the control of concurrently interacting systems. These two reasons also justify the successful use of temporal logic as a specification language in the concurrency and computer aided verification communities [10], [12]. In the next section, we show through simple examples how control specifications can be easily expressed in LTL.

The approach presented in this paper is also an important contribution towards the synthesis of correct by design systems. Temporal logic enables the use of powerful automata theoretic techniques lying at the heart of computational algorithms for 
control design. Transferring control design from the continuous to the finite world of automata (or transition systems) is in fact one of the major contributions of this paper. This transfer is also accompanied by the relevant refinement techniques allowing the transformation of finite automata models of the closed-loop dynamics into hybrid models where software controllers supervise continuous plants. In addition to presenting a fully automated design method, the resulting closed-loop systems satisfy the LTL specifications by construction, therefore resulting in correct designs for which no further validation or verification is necessary.

\section{B. Problem Formulation}

Temporal logic allows one to succinctly describe many interesting temporal properties of systems. LTL formulas are built from predicates through the usual propositional connectives $(\vee, \wedge, \Rightarrow, \neg)$ and two temporal operators: $\circ$ and U. Informally, $\circ$ is read as "next" and a LTL formula $\circ \varphi$ is satisfied when formula $\varphi$ is satisfied at the next time instant. The operator $\mathbf{U}$ is read as "until" and formula $\phi \mathbf{U} \varphi$ is satisfied when formula $\phi$ is satisfied until formula $\varphi$ is satisfied. From the "until" operator, two commonly used operators can be defined: $\square$ and $\diamond$. The first is read as "always," requiring that $\varphi$ holds for all future time in order for $\square \varphi$ to be satisfied. The operator $\diamond$ is read as "eventually" and $\diamond \varphi$ requires $\varphi$ to hold at some time in the future. This set of operators permits the construction of formulas expressing many interesting control specifications which we now illustrate by simple examples.

Periodic synchronization: Consider two mobile robots performing a collaborative task. Each robot is sensing different information that should be shared with the other robot at least every three units of time. We consider robots described by discrete-time linear control systems

$$
\begin{aligned}
& x_{1}(t+1)=A_{1} x_{1}(t)+B_{1} u_{1}(t) \\
& x_{2}(t+1)=A_{2} x_{2}(t)+B_{2} u_{2}(t) .
\end{aligned}
$$

Vector $x_{1} \in \mathbb{R}^{2}$ models the position of robot 1 while vector $x_{2} \in \mathbb{R}^{2}$ models the position of robot 2 . We model the exchange of information between the robots by the requirement that inter robot distance is reduced to less than $\delta>0$ for communication to occur. This distance constraint is captured by the predicate

$$
\text { communicate }:=d\left(x_{1}, x_{2}\right) \leq \delta
$$

for some metric $d$. The desired inter robot communication specification can now be modeled in LTL as

$$
\varphi=\mathcal{S} \diamond_{3} \text { communicate }
$$

where $\diamond_{3}$ is an abbreviation for "eventually within 3 units of time" and is defined by $\diamond_{3} p=p \vee \circ p \vee \circ \circ p \vee \circ \circ \circ p$. Satisfaction of formula $\varphi$ requires that at each time step $\diamond_{3}$ communicate holds, that is, communication will always occur within the next 3 time units.
Path planning and obstacle avoidance: Consider now a robot navigating in an environment cluttered with obstacles. Let $\mathrm{Obs}_{i}$ be a predicate modeling the location of obstacle $i \in I$ and let Goal be a predicate modeling the destination location. Requiring the robot to reach the destination while avoiding the obstacles can be captured by $\diamond$ Goal $\wedge \square \neg\left(\vee_{i \in I} \mathrm{Obs}_{i}\right)$.

Fault tolerance and recovery: Fault tolerance and recovery can also be specified in LTL. Let $\varphi_{1}$ be an LTL formula specifying the normal operation of the system, $\varphi_{2}$ a LTL formula describing the occurrence of a fault and $\varphi_{3}$ an LTL formula prescribing the desired fault recovery procedure. The formula $\square\left(\varphi_{1} \vee\left(\varphi_{1} \mathbf{U}\left(\varphi_{2} \wedge \circ \varphi_{3}\right)\right)\right)$ states that the system should always operate correctly $\left(\varphi_{1}\right)$ or it should operate correctly until $\varphi_{2} \wedge \circ \varphi_{3}$ holds. If this last formula is true, then the fault described by $\varphi_{2}$ occurs at some time $t$ and is followed by the fault recovery procedure, defined by $\varphi_{3}$, at time $t+1$.

The previous examples represent only a small fraction of the interesting properties that can be specified through the use of LTL. The goal of this paper, synthesizing controllers enforcing LTL specifications, can thus be described as follows.

Problem: Let $\Sigma$ be a discrete-time linear control system and $\varphi$ a LTL formula describing the desired behavior for $\Sigma$. Design a controller for $\Sigma$ such that the closed-loop system satisfies $\varphi$.

The solution to the aforementioned problem will require an interesting combination of computer science and control theoretic concepts and methods briefly described in the next section.

\section{Approach and Main Contributions}

The synthesis of controllers enforcing LTL specifications relies on the possibility of extracting finite models from continuous control systems. These finite abstractions will be equivalent (in a precise sense to be defined) to the continuous models therefore enabling the solution of control problems posed for continuous linear systems through discrete algorithmic techniques. Resulting discrete models for the closed-loop system are then refined, resulting in controllers for the original continuous system whose hybrid closed-loop behavior will satisfy the desired specification. The overall approach is pictured in Fig. 1 and organized as follows.

In Section II, we present one of the paper's main contribution. We show that any discrete-time controllable linear system admits finite abstractions (bisimulations) with respect to a certain class of observation functions defined by the system dynamics. The existence of such finite abstractions is one of the essential factors enabling the development of algorithms for system analysis and design. In this paper, we will use finite abstractions of linear control systems to algorithmically synthesize controllers for LTL specifications. This will be done by constructing a finite supervisor for the discrete abstraction enforcing the LTL specification. Since supervisory synthesis is based on operational models such as finite state machines, Büchi automata or Petri nets, in Section IV we introduce LTL and discuss the conversion of LTL formulas into Büchi automata. Supervisory synthesis is the subject of Section V where it is recalled that existence of finite supervisors enforcing infinite languages defined by Büchi automata can be decided and that such supervisors can be effectively computed. In Section V we refine the closed-loop behavior obtained by composing the finite abstraction with the 


\section{Specification Controller Plant}

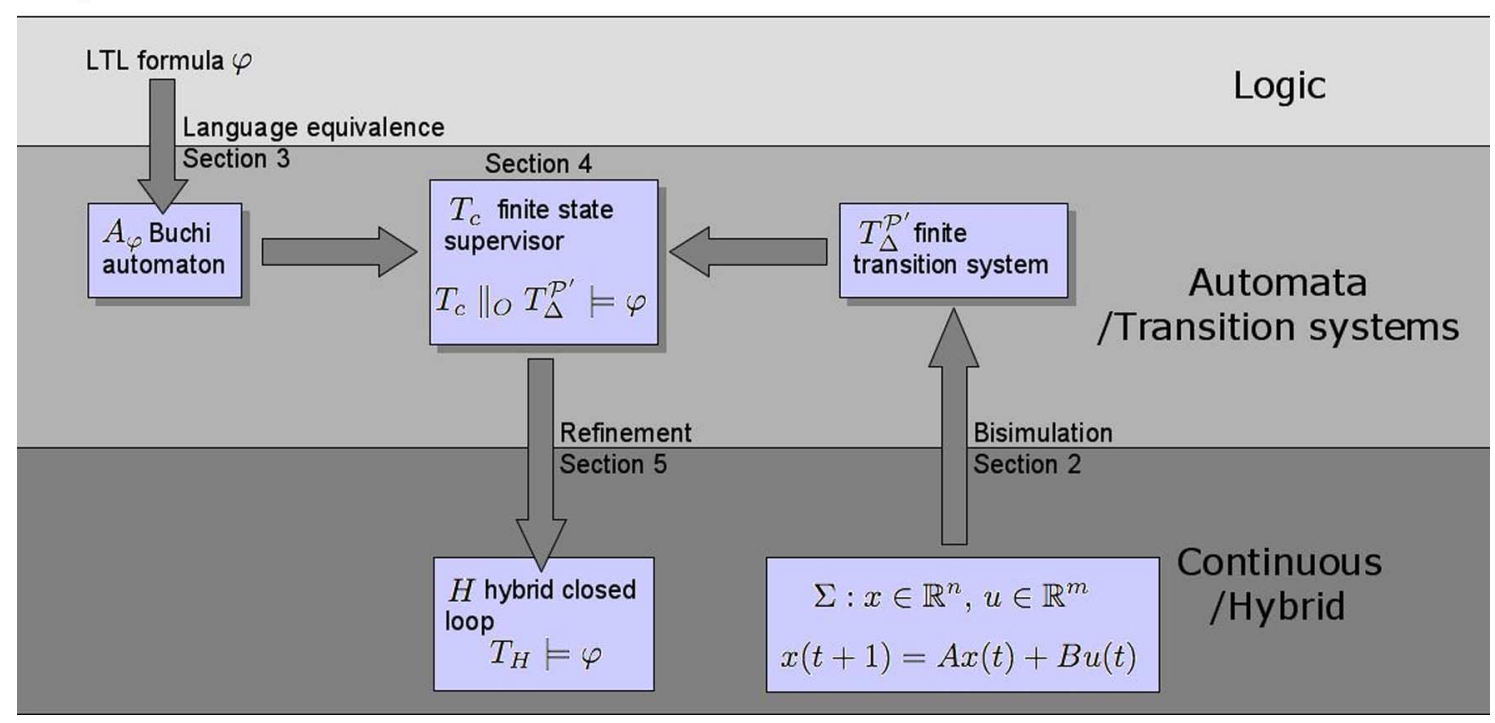

Fig. 1. Intermediate steps in controller design.

discrete supervisor obtaining a hybrid closed-loop behavior enforcing the specification. The other main contribution of the paper is the possibility to synthesize this hybrid controller in a completely automated way. This result is formulated and proved also in Section V. We conclude the paper at Section VI with a discussion of the presented results.

The proposed methodology makes extensive use of both control and computer science concepts, notions and results. Since many of these notions may be unfamiliar to some readers we have decided to focus on the main aspects of the approach, thereby leaving for a later opportunity a more careful discussion of the algorithmic complexity issues as well as the many existing techniques to reduce complexity. We are also not explicitly addressing the centralized/decentralized nature of the resulting controllers, which although important, is a very difficult problem as many important decentralization questions are undecidable [13]. For the same reasons, we have decided to omit large examples and illustrate the introduced notions and algorithms with small, yet pedagogical examples.

\section{Related Literature}

The analysis and synthesis of systems based on temporal logic specifications is by now current practice in the concurrency and computer aided verification communities [10], [12]. Although this approach was initially devised for purely discrete systems, the seminal work of Alur and Dill on timed automata [14] showed that certain classes of hybrid systems could also be addressed. Subsequent extensions lead to results for multi-rate automata [15] and rectangular hybrid automata [16], [17] which lies on the decidability boundary [9]. These results were based on the construction of finite abstractions on which algorithms with guaranteed termination can be used for analysis and synthesis. Different classes of dynamics for which finite abstractions exist were introduced in [18] by combining tools from logic and linear dynamical systems. See also [19] for a survey of these methods. Nonlinear dynamics were considered in [20] where bisimulations based on foliations transverse to the nonlinear flow were introduced. In [21], invariants are also exploited for a supervisory control approach to the control of hybrid systems. A different kind of dynamics, simple planar differential inclusions, was considered in [22] where it was shown that qualitative analysis of system trajectories is decidable by making use of unique topological properties of the plane. Different approaches based on approximation techniques to obtain finite abstractions include the work in [23] for verification and [24] for synthesis of supervisor controllers. Recently, a different abstraction technique based on quantifier elimination was introduced in [25]. This methodology allows one to obtain a sequence of finer finite abstractions that are sufficient to verify reachability related questions.

From the different mentioned approaches, only the work described in [20] address the problem of constructing (exact) finite abstractions of control systems. For linear systems, controllability can be exploited to compute the foliations required by the method in [20] leading to finite abstractions of the vector field obtained by fixing the control inputs. Although at the technical level we do not make use of foliations, our construction can be seen as providing a way of integrating in the same finite object the different abstractions of [20] obtained for different control inputs. However, our construction considers discrete-time systems, while the results in [20] were developed for continuous time.

The construction of finite abstractions is also related to the study of reachability of quantized systems [26], [28]. For quantized systems, the original continuous dynamics is unchanged, but the set of available inputs is restricted to a finite set. This approach also provides an abstraction of the original control system, that can be regarded as a subsystem of the original one. Our approach differs from quantization based reachability in that we do not restrict the set of available inputs. Nevertheless, both approaches emphasize the advantages of having finite representations. Other related work includes the study of stabilization of linear systems with quantized observations [29], [30].

Synthesis of controllers from temporal logic specifications had already been advocated in [31] where the authors postulate a discrete abstraction for the walking mechanism to be controlled. In [32], temporal logic is used to motivate the devel- 
opment of the synthesis procedures as well as to prove several facts regarding the proposed algorithms. Different automated synthesis procedures is reported in [33], where it shown that synthesis of reachability specifications for hybrid systems with linear dynamics on convex polytopes can be performed by simply working with the polytopes vertices. Closer to our approach is the work reported in [34], where it is shown that under certain controllability assumptions the controlled invariance problem for linear systems is decidable. Although our decidability results are also based on a controllability assumption, the problems being addressed are fundamentally different. We refine a given partition of the state space until a bisimulation is obtained while in [34] a set is refined until controlled invariance is achieved. The goal of the refinement algorithms is therefore distinct, although termination is ensured in both cases by controllability.

Other related work, based on supervisory control of discrete event systems [35]-[37], includes synthesis for CTL* specifications [38] and real-time logic [39]. However, synthesis from temporal logic specifications in the computer science community can be traced back to [40], [41]. More recent work includes controller synthesis for branching time specifications [42], decentralized control [43], [44], control of synchronous systems [45], [46] and synthesis for several different problems in timed automata including game theoretic approaches [47], scheduling [48], optimal control [49], [50] and synthesis from external specifications [51]. Although many of these works provide valuable inspiration, the proposed synthesis methodologies are only applicable to purely discrete systems or systems modeled by timed automata.

\section{Finite QuotiEnTS of CONTROLlable LinEAR SystemS}

In this section, we show that finite abstractions of controllable linear systems exist and are effectively computable. These results will make a fundamental use of several computer science notions that we now review.

\section{A. Transition Systems and Bisimulations}

Given a function $f: A \rightarrow B$ and a set $C \subseteq A$, we will use the notation $f(C)$ to denote the subset of $B$ defined by $\cup_{c \in C}\{f(c)\}$ while $f^{-1}(D)$ denotes the set $\{a \in A \mid f(a) \in D\}$ for some $D \subseteq B$. A partition $\mathcal{P}$ of the set $A$ is a collection of sets $\mathcal{P}=\left\{P_{i}\right\}_{i \in I}$ satisfying $\cup_{i \in I} P_{i}=A$ and $P_{i} \cap P_{j} \neq \varnothing$ for $i \neq j$. Each partition induces a projection map $\pi_{\mathcal{P}}: A \rightarrow \mathcal{P}$ sending each $a \in A$ to the unique set $\pi_{\mathcal{P}}(a)=P \in \mathcal{P}$ containing $a$. Conversely, every surjective map $\pi: A \rightarrow B$ defines a partition of $A$ defined by the collection of sets $\left\{\pi^{-1}(b)\right\}_{b \in B}$. An equivalence relation $R \subseteq A \times A$ on a set $A$ induces a partition $\mathcal{P}=\left\{P_{i}\right\}_{i \in I}$ defined by $a, b \in P_{i}$ iff $(a, b) \in R$. The elements $P_{i}$ of the partition $\mathcal{P}$ are the equivalence classes of $R$. Conversely, given a partition $\mathcal{P}$ on $A$ we can define an equivalence relation $R \subseteq A \times A$ having the elements of $\mathcal{P}$ as equivalence classes. For this reason we will interchangeably work with partitions or equivalence relations according to what will be more useful. We say that partition $\mathcal{P}^{\prime}$ refines or that it is a refinement of partition $\mathcal{P}$ when for every $P^{\prime} \in \mathcal{P}^{\prime}$ there exists a $P \in \mathcal{P}$ such that $P^{\prime} \subseteq P$. Given a refinement $\mathcal{P}^{\prime}$ of a partition $\mathcal{P}$ we can define a projection map $\pi_{\mathcal{P}^{\prime} \mathcal{P}}: \mathcal{P}^{\prime} \rightarrow \mathcal{P}$ taking every
$P^{\prime} \in \mathcal{P}$ to the unique element $\pi_{\mathcal{P}^{\prime} \mathcal{P}}\left(P^{\prime}\right)=P \in \mathcal{P}$ such that $P^{\prime} \subseteq P$.

We recall some formal language notions. Given a set $S$ we denote by $S^{*}$ the set of all finite strings obtained by concatenating elements in $S$. An element of $S^{*}$ is, therefore, given by $s_{1} s_{2} \ldots s_{n}$ with $s_{i} \in S$ for $i=1, \ldots, n$. By $S^{\omega}$ we denote the set of all infinite strings obtained by concatenating elements in $S$. An element of $S^{\omega}$ is an infinite string $s_{1} s_{2} s_{3} \ldots$ with $s_{i} \in S, i \in \mathbb{N}$. Given a string $s$ belonging to $S^{*}$ or $S^{\omega}$ we denote by $s(i)$ the $i$ th element of $s$. The length of a string $s \in S^{*}$ is denoted by $|s|$. A subset of $S^{*}$ is called a language while a subset of $S^{\omega}$ is called an $\omega$-language.

We also review the notion of transition systems that will be extensively used as an abstract model for control and computation.

Definition 2.1: A transition system with observations is a tuple $T=\left(Q, Q^{0}, \longrightarrow, O, \Upsilon\right)$ where

- $Q$ is a (possibly infinite) set of states;

- $Q^{0} \subseteq Q$ is a set of initial states;

- $\longrightarrow \subseteq Q \times Q$ is a transition relation;

- $O$ is a (possibly infinite) set of observations;

- $\Upsilon: Q \rightarrow O$ is a map assigning to each $q \in Q$ an observation $\Upsilon(q) \in O$.

A string $s \in Q^{*} \cup Q^{\omega}$ is a run of $T$ if $(s(i), s(i+1)) \in \longrightarrow, i=$ $1, \ldots,|s|-1$ for $s \in Q^{*}$ or $i \in \mathbb{N}$ for $s \in Q^{\omega}$. A run of $T$ is initialized when $s(1) \in Q^{0}$.

The introduced notion of transition system differs from other notions encountered in the literature in that observations are not associated with transitions but rather with states. These two models can easily be seen equivalent given the well known equivalence between Moore and Mealy machines [25]. The presented model is, however, more natural since observations of control systems depend on the states and this structure is inherited by the several transition systems used in this paper to capture the dynamics of control systems.

We say that $T$ is finite when $Q, O$ are finite, and infinite otherwise. We will usually denote by $q \longrightarrow q^{\prime}$ a pair $\left(q, q^{\prime}\right)$ belonging to $\longrightarrow$. As we will only consider transition systems with observations, we shall refer to them simply as transition systems. Since the observation map $\Upsilon: Q \rightarrow O$ extends to a unique map of strings $\Upsilon: Q^{*} \cup Q^{\omega} \rightarrow O^{*} \cup O^{\omega}$ defined by

$$
\Upsilon(s)=\Upsilon(s(1)) \Upsilon(s(2)) \Upsilon(s(3)) \ldots
$$

we will abuse notation and use the same symbol $\Upsilon$ for both the observation map as well as for its induced string map. Given a state $q \in Q$, we denote by $\operatorname{Pre}(q)$ the set of states in $Q$ that can reach $q$ in one step, that is

$$
\operatorname{Pre}(q)=\left\{q^{\prime} \in Q \mid q^{\prime} \longrightarrow q\right\} .
$$

We extend Pre to sets $Q^{\prime} \subseteq Q$ in the usual way

$$
\operatorname{Pre}\left(Q^{\prime}\right)=\bigcup_{q^{\prime} \in Q^{\prime}} \operatorname{Pre}\left(q^{\prime}\right)
$$


Discrete-time linear control systems can be naturally embedded in the class of transition systems. Given a discrete-time linear control system $\Sigma$

$$
x(t+1)=A x(t)+B u(t), \quad x \in \mathbb{R}^{n}, \quad u \in \mathbb{R}^{m}, \quad t \in \mathbb{N}
$$

there is an associated transition system $T_{\Sigma}$

$$
T_{\Sigma}=\left(\mathbb{R}^{n}, \mathbb{R}^{n}, \longrightarrow, O, \Upsilon\right)
$$

with $\longrightarrow \subseteq \mathbb{R}^{n} \times \mathbb{R}^{n}$ defined by $x \longrightarrow x^{\prime}$ iff there exists a $u \in \mathbb{R}^{m}$ such that $x^{\prime}=A x+B u$. To complete the definition of $T_{\Sigma}$ we must also provide an observation set $O$ and observation map $\Upsilon$. The nature of the observation space and map depend on the problem being solved and are left unspecified for now. The described embedding is control abstract since the input value required to perform transition $x \longrightarrow x^{\prime}$ is not explicitly captured by the transition system. However, this information can be recovered from the pair $\left(x, x^{\prime}\right)$ by solving $x^{\prime}=A x+B u$ for the input $u$. Since transition systems capture both control systems and software systems, we can synthesize controllers consisting of continuous and discrete (components) within the same framework.

Transition systems define different types of languages.

Definition 2.2: Let $T=\left(Q, Q^{0}, \longrightarrow, O, \Upsilon\right)$ be a transition system. The language generated by $T$, denoted by $L(T)$, is defined as:

$$
\left\{r \in O^{*} \mid r=\Upsilon(s) \text { for some finite initialized run } s \text { of } T\right\} \text {. }
$$

The $\omega$-language generated by $T$ is similarly defined:

$\left\{r \in O^{\omega} \mid r=\Upsilon(s)\right.$ for some infinite initialized run $s$ of $\left.T\right\}$.

The structural notion of bisimulation relates properties of different transition systems.

Definition 2.3: Let $T_{i}=\left(Q_{i}, Q_{i}^{0}, \longrightarrow_{i}, O, \Upsilon_{i}\right)$ with $i=1,2$ be transition systems and $R \subseteq Q_{1} \times Q_{2}$ a relation. Relation $R$ is said to be a bisimulation relation between $T_{1}$ and $T_{2}$ if the following hold for any $\left(q_{1}, q_{2}\right) \in R$ :

- $\Upsilon_{1}\left(q_{1}\right)=\Upsilon_{2}\left(q_{2}\right)$;

- $q_{1} \in Q_{1}^{0}$ implies $q_{2} \in Q_{2}^{0}$ and $q_{2} \in Q_{2}^{0}$ implies $q_{1} \in Q_{1}^{0}$;

- $q_{1} \longrightarrow 1 q_{1}^{\prime}$ implies the existence of $q_{2}^{\prime} \in Q_{2}$ satisfying $q_{2} \longrightarrow_{2} q_{2}^{\prime}$ and $\left(q_{1}^{\prime}, q_{2}^{\prime}\right) \in R$;

- $q_{2} \longrightarrow_{2} q_{2}^{\prime}$ implies the existence of $q_{1}^{\prime} \in Q_{1}$ satisfying $q_{1} \longrightarrow q_{1}^{\prime}$ and $\left(q_{1}^{\prime}, q_{2}^{\prime}\right) \in R$.

We shall use the notation $T_{1} \cong T_{2}$ to denote the existence of a bisimulation relation between $T_{1}$ and $T_{2}$. Bisimilar transition systems share many properties including generated languages.

Proposition 2.4 (Adapted from [53]): Let $T_{1}$ and $T_{2}$ be transition systems and assume that $T_{1} \cong T_{2}$. Then, the following equalities hold:
In addition to preserve language equivalence, bisimulations also preserve properties expressible in several temporal logics such as LTL, CTL, CTL ${ }^{*}$ or $\mu$-calculus [54].

In this paper, we will construct finite bisimulations which are of a special form.

Definition 2.5: The quotient of a transition system $T=\left(Q, Q^{0}, \longrightarrow, O, \Upsilon\right)$ with respect to an equivalence relation $R \subseteq Q \times Q$ is the transition system $T_{/ R}=\left(Q_{/ R}, Q_{/ R}^{0}, \longrightarrow / R, O, \Upsilon_{/ R}\right)$ defined by

- $Q_{/ R}=\{S \subseteq Q \mid S$ is an equivalence class of $R\}$;

- $Q_{/ R}^{0}=\pi_{R}\left(Q^{0}\right)$;

- $S \longrightarrow / R S^{\prime}$ in $T_{/ R}$ if there exists $q \in S$ and $q^{\prime} \in S^{\prime}$ such that $q \longrightarrow q^{\prime}$ in $T$;

- $\Upsilon_{/ R}(S)=\Upsilon(q)$ for some $q \in S$.

Note that $\Upsilon_{/ R}$ is well defined since $\left(q_{1}, q_{2}\right) \in R$ implies $\Upsilon\left(q_{1}\right)=\Upsilon\left(q_{2}\right)$. Furthermore, if $R$ is a bisimulation relation between $T$ and $T$ it follows that the graph of the projection $\pi_{R}: Q \rightarrow Q_{/ R}$, defined by $\left\{(q, S) \in Q \times Q_{R} \mid S=\pi_{R}(q)\right\}$, is a bisimulation relation between $T$ and $T_{/ R} . T_{/ R}$ is, therefore, called a bisimilar quotient of $T$ with respect to $R$.

\section{B. Finite Bisimulations of Controllable Linear Systems}

In this section, we show how finite bisimulations of controllable linear systems can be obtained. We make the following assumptions.

A.I) Control system $\Sigma$ is controllable.

A.II) The columns of matrix $B$ are linearly independent.

Assumption A.II) results in no loss of generality since we can always remove linearly dependent columns from matrix $B$ without destroying essential properties of $\Sigma$. Assumption A.I) is essential for the existence of finite bisimulations. It has several important consequences, the first of which being the following decomposition of the state-space.

Proposition 2.6 [55], [56]: Let $\Sigma$ be a discrete-time linear control system satisfying Assumptions A.I) and A.II). Then, there exists a sequence of positive integers $\mu_{1}, \mu_{2}, \ldots, \mu_{m}$, called controllability indexes of $\Sigma$, such that:

$\operatorname{span}\left\{b_{1}, \ldots, b_{m}, A b_{1}, \ldots\right.$

$$
\left.A b_{m}, \ldots, A^{n-1} b_{1}, \ldots, A^{n-1} b_{m}\right\}
$$

equals

$$
\begin{array}{r}
\operatorname{span}\left\{b_{1}, A b_{1}, \ldots, A^{\mu_{1}-1} b_{1}, b_{2}, A b_{2}, \ldots, A^{\mu_{2}-1} b_{2}, \ldots\right. \\
\left.b_{m}, A b_{m}, \ldots, A^{\mu_{m}-1} b_{m}\right\}
\end{array}
$$

and $A^{\mu_{i}} b_{i}$ is linearly dependent of the vectors $b_{1}, A b_{1}, \ldots, b_{i}, A b_{i}, \ldots, A^{\mu_{i}-1} b_{i}$.

Using the controllability indices we can introduce the subspace $V \cong \mathbb{R}^{n-m}$ of $\mathbb{R}^{n}$ defined by

$$
\begin{array}{r}
\operatorname{span}\left\{b_{1}, \ldots, A^{\mu_{1}-2} b_{1}, b_{2}, \ldots, A^{\mu_{2}-2} b_{2}, \ldots\right. \\
\left.b_{m}, \ldots, A^{\mu_{m}-2} b_{m}\right\} .
\end{array}
$$


Subspace $V$ naturally induces an observation map $\Upsilon$ for $\Sigma$ defined as the natural projection from the state-space $\mathbb{R}^{n}$ to the quotient space $\mathbb{R}^{n} / V \cong \mathbb{R}^{m}$

$$
\Upsilon: \mathbb{R}^{n} \rightarrow \mathbb{R}^{n} / V
$$

Observation map $\Upsilon$ takes a vector $x \in \mathbb{R}^{n}$ into its equivalence class in $\mathbb{R}^{n} / V$ which we can identify with a point $y \in \mathbb{R}^{m}$. Observation map $\Upsilon$ uniquely determines transition system $T_{\Sigma}$ associated with $\Sigma$. We now state this fact for later use.

Definition 2.7: Let $\Sigma$ be a discrete-time linear control system satisfying Assumptions A.I) and A.II). Transition system $T_{\Sigma}$ associated with $\Sigma$ is defined by $T_{\Sigma}=\left(\mathbb{R}^{n}, \mathbb{R}^{n}, \longrightarrow, \mathbb{R}^{m}, \Upsilon\right)$ with $x \longrightarrow x^{\prime}$ iff there exists $u \in \mathbb{R}^{m}$ satisfying $x^{\prime}=A x+B u$ and $\Upsilon$ defined by (2).

This choice of observation map is crucial in proving the first major contribution of this paper.

Theorem 2.8: Let $\Sigma$ be a discrete-time linear control system satisfying Assumptions A.I) and A.II). For any finite partition $\mathcal{P}$ of the observation space of $T_{\Sigma}$ there exists a finite refinement $\mathcal{P}^{\prime}$ of the state space partition $\Upsilon^{-1}(\mathcal{P})$ such that the quotients of $T_{\Sigma}^{\mathcal{P}}=\left(\mathbb{R}^{n}, \mathbb{R}^{n}, \longrightarrow, \mathcal{P}, \pi_{\mathcal{P}} \circ \Upsilon\right)$ and $T_{\Sigma}^{\mathcal{P}^{\prime}}=\left(\mathbb{R}^{n}, \mathbb{R}^{n}, \longrightarrow\right.$ $\left., \mathcal{P}^{\prime}, \pi_{\mathcal{P}^{\prime}}\right)$ with respect to $\mathcal{P}^{\prime}$ and denoted by $T_{\Delta}^{\mathcal{P}}$ and $T_{\Delta}^{\mathcal{P}^{\prime}}$, respectively, are finite bisimilar quotients.

In order to prove Theorem 2.8 , we state and prove a preparatory result ensuring that existence of finite bisimulations is not destroyed by changes of coordinates or invertible feedback.

Proposition 2.9: Let $\Sigma$ be a discrete-time linear control system satisfying Assumptions A.I) and A.II), and let $\Sigma^{\prime}$ be the discrete-time linear control system obtained from $\Sigma$ through an invertible linear change of coordinates $x \mapsto H x$ and an invertible linear feedback $(x, u) \mapsto F x+G u$. For any finite partition $\mathcal{P}$ of the observation space of $T_{\Sigma}$, there exists a finite refinement $\mathcal{Q}$ of the state space partition $\Upsilon^{-1}(\mathcal{P})$ making the quotient of $T_{\Sigma}$ with respect to $\mathcal{Q}$ a finite bisimilar quotient iff there exists a finite refinement $\mathcal{Q}^{\prime}$ of the state-space partition $\Upsilon^{\prime-1}(H(\mathcal{P}))$ making the quotient of $T_{\Sigma^{\prime}}$ with respect to $\mathcal{Q}^{\prime}$ a finite bisimilar quotient.

Proof: Assume that $\mathcal{Q}$ exists and let $\mathcal{Q}^{\prime}$ be the finite partition $H(\mathcal{Q})$ of the state space of $T_{\Sigma^{\prime}}$ (note that $H(\mathcal{Q})$ is a partition since $H$ is an invertible matrix). It is clear that $\mathcal{Q}^{\prime}$ refines $\Upsilon^{\prime-1}(H(\mathcal{P}))$. To show that $\mathcal{Q}^{\prime}$ is a bisimulation relation between $T_{\Sigma^{\prime}}$ and $T_{\Sigma^{\prime}}$ consider $\left(z_{1}, w_{1}\right) \in \mathcal{Q}^{\prime}$ and assume that $z_{1} \longrightarrow z_{2}$ in $T_{\Sigma^{\prime}}$. By definition of $\mathcal{Q}^{\prime}$ and invertibility of $H$, there exists $\left(x_{1}, y_{1}\right) \in \mathcal{Q}$ such that $\left(H x_{1}, H y_{1}\right)=\left(z_{1}, w_{1}\right)$ and $x_{1} \longrightarrow x_{2}$ in $T_{\Sigma}$ with $H x_{2}=z_{2}$. It then follows from the fact that $\mathcal{Q}$ is a bisimulation relation between $T_{\Sigma}$ and $T_{\Sigma}$ that $y_{1} \longrightarrow y_{2}$ in $T_{\Sigma}$ with $\left(x_{2}, y_{2}\right) \in \mathcal{Q}$. Let $u$ be the input triggering the transition $y_{1} \longrightarrow y_{2}$. Then, input $F y_{1}+G u$ triggers a transition $H y_{1}=w_{1} \longrightarrow w_{2}=H y_{2}$ in $T_{\Sigma}^{\prime}$ and $\left(z_{2}, w_{2}\right) \in \mathcal{Q}^{\prime}$ since $\left(z_{2}, w_{2}\right)=\left(H x_{2}, H y_{2}\right)$ and $\left(x_{2}, y_{2}\right) \in \mathcal{Q}$. This proves condition (3) in Definition 2.3 and condition (4) is proved using the same argument. Condition (2) is trivially satisfied since the set of initial states is $\mathbb{R}^{n}$ and $H$ is invertible while condition (1) follows from the equality $\Upsilon=\Upsilon^{\prime} \circ H$.

We now return to the proof of Theorem 2.8.

Proof (of Theorem 2.8): In view of Proposition 2.9 we can assume, without loss of generality, that $\Sigma$ is in Brunovsky normal form since any controllable linear system can be transformed into this form by a change of coordinates and an invertible feedback [55], [56]. Recall that the Brunovsky normal form of a controllable linear system with controllability indexes $\mu_{1}, \ldots, \mu_{m}$ is given by

$$
\begin{aligned}
& x_{1}(t+1)=x_{2}(t) \quad x_{\mu_{1}+1}(t+1)=x_{\mu_{1}+2}(t) \\
& x_{2}(t+1)=x_{3}(t) \quad x_{\mu_{1}+2}(t+1)=x_{\mu_{1}+3}(t) \\
& x_{\mu_{1}}(t+1)=u_{1}(t) \quad x_{\mu_{1}+\mu_{2}}(t+1)=u_{2}(t) \\
& \ldots \quad x_{\mu_{1}+\cdots+\mu_{m-1}+1}(t+1)=x_{\mu_{1}+\cdots+\mu_{m-1}+2}(t) \\
& \ldots \quad x_{\mu_{1}+\cdots+\mu_{m-1}+2}(t+1)=x_{\mu_{1}+\cdots+\mu_{m-1}+3}(t) \\
& : \\
& \ldots \quad x_{\mu_{1}+\cdots+\mu_{m}}(t+1)=u_{m}(t) .
\end{aligned}
$$

A simple computation shows that for a control system in Brunovsky normal form $\Upsilon$ is of the form

$$
\Upsilon=\phi \circ \pi
$$

where $\pi: \mathbb{R}^{n} \rightarrow \mathbb{R}^{m}$ is the projection map $\pi(x)=$ $\left(x_{1}, x_{\mu_{1}+1}, \ldots, x_{\mu_{1}+\cdots+\mu_{m-1}+1}\right)$ and $\phi$ is an arbitrary linear isomorphism. We will now introduce some notation to simplify the proof. We will use $x(t+i, u(t), u(t+1), \ldots, u(t+i-1))$ to denote $x(t+i)$ and to emphasize that $x(t+i, u(t), u(t+$ $1), \ldots, u(t+i-1))$ is obtained from $x(t)$ by applying the sequence of inputs $u(t), u(t+1), \ldots, u(t+i-1)$. We will also denote by $\mu$ the largest controllability index.

We now note that it follows from (5) that input $u_{k}(t+j)$ will not affect $\Upsilon(x(t+i, u(t), \ldots, u(t+i-1)))$ when $\mu_{k}>i-j$. In other words, $\mu_{k}>i-j$ implies

$$
\frac{\partial}{\partial u_{k}(t+j)} \Upsilon(x(t+i), u(t), \ldots, u(t+i-1))=0 .
$$

To illustrate this remark, consider a control system defined by $x_{1}(t+1)=x_{2}(t)$ and $x_{2}(t+1)=u(t)$ with observation map $\Upsilon(x)=x_{1}$. Since the controllability index corresponding to input $u$ is 2 we see that for $j=0$

$$
\begin{aligned}
(i=0) \quad \frac{\partial}{\partial u(t)} \Upsilon(x(t)) & =\frac{\partial}{\partial u(t)} x_{1}(t)=0 \\
(i=1) \quad \frac{\partial}{\partial u(t)} \Upsilon(x(t+1, u(t))) & =\frac{\partial}{\partial u(t)} x_{2}(t)=0 .
\end{aligned}
$$

This remark will be used several times in the proof.

Consider now the equivalence relation $R^{k} \subseteq \mathbb{R}^{n} \times \mathbb{R}^{n}$ recursively defined as follows:

$$
\begin{array}{r}
(x(t), y(t)) \in R^{0} \text { iff } \pi_{\mathcal{P}} \circ \Upsilon(x(t))=\pi_{\mathcal{P}} \circ \Upsilon(y(t)) \\
(x(t), y(t)) \in R^{k+1} \text { iff } \forall u(t) \in \mathbb{R}^{m} \exists v(t) \in \mathbb{R}^{m} \\
\wedge \forall v(t) \in \mathbb{R}^{m} \exists u(t) \in \mathbb{R}^{m} \\
(x(t+1, u(t)), y(t+1, v(t))) \in R^{k} .
\end{array}
$$

We claim that $R^{\mu-1}$ is an auto-bisimulation relation. Since condition (1) in Definition 2.3 follows from the chosen observation function for $T_{\Sigma}^{\mathcal{P}}$ and $T_{\Sigma}^{\mathcal{P}^{\prime}}$ and condition (2) follows from the equality $Q_{0}=\mathbb{R}^{n}$, in order to prove the claim we only need to show that for any $(x(t), y(t)) \in R^{\mu-1}$ 
1) if $x(t) \longrightarrow x(t+1, u(t))$, then there exists $y(t+1, v(t))$ satisfying $y(t) \longrightarrow y(t+1, v(t))$ and $(x(t+1, u(t)), y(t+$ $1, v(t))) \in R^{\mu-1}$

2) if $y(t) \longrightarrow y(t+1, v(t))$, then there exists $x(t+1, u(t))$ satisfying $x(t) \longrightarrow x(t+1, u(t))$ and $(x(t+1, u(t)), y(t+$ $1, v(t))) \in R^{\mu-1}$.

We will show only (1) since the same argument is valid for (2). We will prove (1) by showing that $(x(t), y(t)) \in R^{\mu-1}$ implies $(x(t), y(t)) \in R^{\mu}$ since (1) would then follow from the definition of $R^{k}$.

Consider then $(x(t), y(t)) \in R^{\mu-1}$ and let $x(t) \longrightarrow x(t+$ $1, u(t))$. It follows from $(x(t), y(t)) \in R^{\mu-1}$ and the definition of $R^{k}$, the existence of $y(t) \longrightarrow y(t+1, v(t))$ satisfying $(x(t+$ $1, u(t)), y(t+1, v(t))) \in R^{\mu-2}$. Making use of (6) for $j=0$ we see that if we modify $v(t)$ to $\bar{v}(t)$ by changing the components $v_{k}(t)$ of $v(t)$ such that $\mu-1<\mu_{k}$ we will still have $(x(t+$ $1, u(t)), y(t+1, \bar{v}(t))) \in R^{\mu-2}$ since $R^{\mu-2}$ is based on the equalities

$$
\begin{aligned}
& \pi_{\mathcal{P}} \circ \Upsilon(x(t+i, u(t), \ldots, u(t+i-1))) \\
& \quad=\pi_{\mathcal{P}} \circ \Upsilon(y(t+i, v(t), \ldots, v(t+i-1)))
\end{aligned}
$$

for $1 \leq i \leq \mu-1$. Thus, we define $\bar{v}(t)$ as

$$
\begin{array}{ll}
\bar{v}_{k}(t)=u_{k}(t), & \text { if } \mu-1<\mu_{k} \\
\bar{v}_{k}(t)=v_{k}(t), & \text { if } \mu-1 \geq \mu_{k} .
\end{array}
$$

At this point we have $(x(t+1, u(t)), y(t+1, \bar{v}(t))) \in R^{\mu-2}$ and we consider $x(t+1, u(t)) \longrightarrow x(t+2, u(t), u(t+1))$. It follows from $(x(t+1, u(t)), y(t+1, \bar{v}(t))) \in R^{\mu-2}$ and the definition of $R^{k}$, the existence of $y(t+1, \bar{v}(t)) \longrightarrow y(t+2, \bar{v}(t), v(t+1))$ satisfying $(x(t+2, u(t), u(t+1)), y(t+2, \bar{v}(t), v(t+1))) \in$ $R^{\mu-3}$. Making use of (6) for $j=1$ we see that if we modify $v(t+1)$ to $\bar{v}(t+1)$ by changing the components $v_{k}(t+1)$ of $v(t+1)$ such that $\mu-2<\mu_{k}$ we will still have $(x(t+$ $2, u(t), u(t+1)), y(t+2, \bar{v}(t), \bar{v}(t+1))) \in R^{\mu-3}$ since $R^{\mu-3}$ is based on the equalities

$$
\begin{aligned}
& \pi_{\mathcal{P}} \circ \Upsilon(x(t+i, u(t), \ldots, u(t+i-1))) \\
= & \pi_{\mathcal{P}} \circ \Upsilon(y(t+i, v(t), \ldots, v(t+i-1)))
\end{aligned}
$$

for $2 \leq i \leq \mu-1$. We thus define $\bar{v}(t+1)$ as

$$
\begin{array}{ll}
\bar{v}_{k}(t+1)=u_{k}(t+1), & \text { if } \quad \mu-2<\mu_{k} \\
\bar{v}_{k}(t+1)=v_{k}(t+1), & \text { if } \quad \mu-2 \geq \mu_{k} .
\end{array}
$$

If we keep on modifying $v$ to $\bar{v}$ according to the previously described process, we will obtain

$$
\begin{aligned}
& (x(t+\mu-1, u(t), \ldots, u(t+\mu-2)) \\
& y(t+\mu-1, v(t), \ldots, v(t+\mu-2))) \in R^{0} .
\end{aligned}
$$

Consider now a transition from $x(t+\mu-1, u(t), \ldots, u(t+\mu-$ $2))$ to $x(t+\mu, u(t), \ldots, u(t+\mu-1))$ and let $\bar{v}(t+\mu-1)$ be an arbitrary element of $\mathbb{R}^{m}$. It then follows the existence of a transition from $y(t+\mu-1, \bar{v}(t), \ldots, \bar{v}(t+\mu-2))$ to $y(t+$ $\mu, \bar{v}(t), \ldots, \bar{v}(t+\mu-1))$ and, furthermore

$$
\begin{aligned}
& \pi(x(t+\mu, u(t), \ldots, u(t+\mu-1))) \\
& \quad=\left(u_{1}\left(t+\mu-\mu_{1}\right), \ldots, u_{m}\left(t+\mu-\mu_{m}\right)\right)
\end{aligned}
$$

$$
\begin{aligned}
& =\left(\bar{v}_{1}\left(t+\mu-\mu_{1}\right), \ldots, \bar{v}_{m}\left(t+\mu-\mu_{m}\right)\right) \\
& =\pi(y(t+\mu, \bar{v}(t), \ldots, \bar{v}(t+\mu-1))
\end{aligned}
$$

in virtue of the way we defined $\bar{v}$ for $0 \leq i \leq \mu-2$. We thus conclude from (5) that

$$
\begin{aligned}
& (x(t+\mu, u(t), \ldots, u(t+\mu-1)) \\
& \quad y(t+\mu, \bar{v}(t), \ldots, \bar{v}(t+\mu-1))) \in R^{0}
\end{aligned}
$$

which, in turn, implies $(x(t), y(t)) \in R^{\mu}$ and concludes the proof of the claim.

To conclude the proof of the theorem we must show that $R^{\mu-1}$ has a finite number of equivalence classes. However, this follows at once from the observation that $R^{\mu-1}$ represents bisimilarity restricted to $\mu-1$ steps and defined with respect to a finite observation space.

The possibility of synthesizing controllers enforcing LTL specifications hinges on Theorem 2.8 as it guarantees that the behavior of $T_{\Sigma}$ admits a finite representation in the form of a bisimilar quotient. This bisimulation is based on a finite partition of the observation space of $T_{\Sigma}$ used to describe the control objectives through a LTL formula. We thus see that the observation space and map of $T_{\Sigma}$, naturally defined by the system dynamics under Assumption II, are essential ingredients of Theorem 2.8.

Since existence of finite bisimulations has been established, the following well known bisimulation algorithm can be used to compute the coarsest possible bisimulation [57], [58] provided that every set operation is effectively computable, that is, provided that there exists an algorithm for a Touring machine implementing the desired set operations. The bisimulation algorithm starts with a transition system $T_{\Sigma}$ and the initial partition $\Upsilon^{-1}(\mathcal{P})$ of $\mathbb{R}^{n}$ and terminates with the coarsest partition $\mathcal{P}^{\prime}$ such that $T_{/ \mathcal{P}^{\prime}}$ is a bisimilar quotient of $T$.

Algorithm 2.10: (Bisimulation Algorithm)

$$
\begin{aligned}
& \text { set }: \mathcal{P}^{\prime}=\Upsilon^{-1}(\mathcal{P}) \\
& \text { while }: \exists P, P^{\prime} \in \mathcal{P}^{\prime} \text { such that } \varnothing \neq P \cap \operatorname{Pre}\left(P^{\prime}\right) \neq P \\
& \quad \text { set }: P_{1}=P \cap \operatorname{Pre}\left(P^{\prime}\right) P_{2}=P \cap \overline{\operatorname{Pre}\left(P^{\prime}\right)} \\
& \quad \text { refine }: \mathcal{P}^{\prime}=\left(\mathcal{P}^{\prime} \backslash\{P\}\right) \cup\left\{P_{1}, P_{2}\right\} \\
& \text { end while. }
\end{aligned}
$$

As we are considering linear control systems it is natural to consider partitions of the observation space $\mathbb{R}^{m}$ defined by semi-linear sets. To ensure computability we restrict all the coefficients to live in $\mathbb{Q}$.

Definition 2.11: The class of semi-linear subsets of $\mathbb{R}^{m}$ consists of finite unions, intersections and complements of the following elementary sets:

$\left\{x \in \mathbb{R}^{m} \mid f^{T} x+c \sim 0, \quad f \in \mathbb{Q}^{m}, \quad c \in \mathbb{Q}, \quad \sim \in\{=,>\}\right\}$.

Computability of the finite bisimulation is now a consequence of effective computability of intersections, unions and complements of semi-linear sets and the fact that Pre of a semi-linear set can be computed by quantifier elimination [59], resulting in a semi-linear set. We thus have the following corollary to Theorem 2.8. 


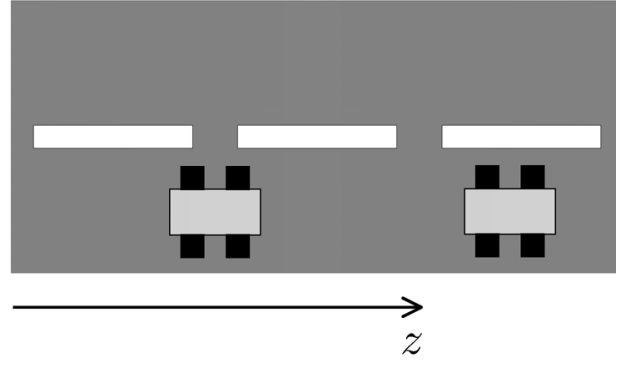

Fig. 2. Identical vehicles following the same lane.

Corollary 2.12: Let $\Sigma$ be a discrete-time linear control system satisfying Assumptions A.I) and A.II) and defined by matrices $A$ and $B$ with rational entries. For any finite partition $\mathcal{P}$ of the observation space of $T_{\Sigma}$ defined by semi-linear sets, the quotients of $T_{\Sigma}^{\mathcal{P}}=\left(\mathbb{R}^{n}, \mathbb{R}^{n}, \longrightarrow, \mathcal{P}, \pi_{\mathcal{P}} \circ \Upsilon\right)$ and $T_{\Sigma}^{\mathcal{P}^{\prime}}=\left(\mathbb{R}^{n}, \mathbb{R}^{n}, \longrightarrow, \mathcal{P}^{\prime}, \pi_{\mathcal{P}^{\prime}}\right)$ with respect to partition $\mathcal{P}^{\prime}$, whose existence is asserted by Theorem 2.8, are finite bisimilar quotients which are effectively computable.

Example 2.13: We now illustrate Theorem 2.8 on a variation of the periodic synchronization example discussed in the introduction. Consider two identical vehicles moving on the same lane as shown in Fig. 2.

For our purposes it will be sufficient to consider the translational dynamics along the lane. Each vehicle is modeled as a discrete-time double integrator

$$
\begin{aligned}
& z_{i}(t+1)=v_{i}(t) \\
& v_{i}(t+1)=u_{i}(t)
\end{aligned}
$$

where $i \in\{1,2\}$ and $z_{i}$ represents the translational position of car $i$. Since we will only be interested in controlling the spacing between the vehicles, we introduce new variables:

$$
y_{1}=z_{2}-z_{1} \quad y_{2}=v_{2}-v_{1} \quad u=u_{2}-u_{1}
$$

leading to the following model:

$$
\begin{aligned}
& y_{1}(t+1)=y_{2}(t) \\
& y_{2}(t+1)=u(t)
\end{aligned}
$$

governing intervehicle spacing measured by $y_{1}$. Observation map $\Upsilon: \mathbb{R}^{2} \rightarrow \mathbb{R}$ satisfies $\Upsilon B=0$ and if we model $\Upsilon$ by the row matrix $[a b]$ we obtain

$$
0=\Upsilon B=\left[\begin{array}{ll}
a & b
\end{array}\right]\left[\begin{array}{l}
0 \\
1
\end{array}\right]=b .
$$

To make the discussion concrete we take $a=1$ leading to $\Upsilon y=y_{1}$ and note that other choices for $a$ would equality work. The requirement that vehicles should come together (in order to communicate) at least every $3 \mathrm{~s}$ can be modeled by

$$
\begin{aligned}
\varphi & =\square \diamond_{3}\left(p_{1} \wedge p_{2}\right) \\
p_{1} & =y_{1}+1 / 2>0 \\
p_{2} & =-y_{1}+1 / 2>0 .
\end{aligned}
$$

Note that predicates $p_{1}$ and $p_{2}$ represent regions on the observation space $\mathbb{R} \cong \mathbb{R}^{2} / V=\mathbb{R}^{2} / \operatorname{span}\{B\}$. The formula $p_{1} \wedge p_{2}$ is satisfied when the distance between vehicles is smaller than 1 and $\square \diamond_{3}\left(p_{1} \wedge p_{2}\right)$ ensures that such distance constraint is satisfied every 3 time steps.

If we denote by $S_{1}$ the set defined by $p_{1} \wedge p_{2}$ and by $S_{2}$ its complement, that is

$$
\begin{aligned}
& S_{1}=\left\{\left(y_{1}, y_{2}\right) \in \mathbb{R}^{2} \mid-\frac{1}{2}<y_{1}<\frac{1}{2}\right\} \\
& S_{2}=\left\{\left(y_{1}, y_{2}\right) \in \mathbb{R}^{2} \mid y_{1} \leq-\frac{1}{2} \vee y_{1} \geq \frac{1}{2}\right\}
\end{aligned}
$$

we can use the bisimulation algorithm with the initial partition $\Pi=\left\{S_{1}, S_{2}\right\}$. Following Algorithm 2.10, we compute:

$$
\begin{aligned}
\operatorname{Pre}\left(S_{1}\right)= & \left\{\left(y_{1}, y_{2}\right) \in \mathbb{R}^{2} \mid-\frac{1}{2}<y_{2}<\frac{1}{2}\right\} \\
S_{2} \cap \operatorname{Pre}\left(S_{1}\right)= & \left\{\left(y_{1}, y_{2}\right) \in \mathbb{R}^{2} \mid\left(y_{1} \leq-\frac{1}{2} \vee y_{1} \geq \frac{1}{2}\right)\right. \\
& \left.\wedge-\frac{1}{2}<y_{2}<\frac{1}{2}\right\} .
\end{aligned}
$$

Since $S_{2} \cap \operatorname{Pre}\left(S_{1}\right) \neq \varnothing$ and $S_{2} \cap \operatorname{Pre}\left(S_{1}\right) \neq S_{2}$ we split $S_{2}$ into the sets $S_{21}$ and $S_{22}$ defined by

$$
\begin{aligned}
S_{21}= & S_{2} \cap \operatorname{Pre}\left(S_{1}\right) \\
= & \left\{\left(y_{1}, y_{2}\right) \in \mathbb{R}^{2} \mid\left(y_{1} \leq-\frac{1}{2} \vee y_{1} \geq \frac{1}{2}\right)\right. \\
& \left.\wedge-\frac{1}{2}<y_{2}<\frac{1}{2}\right\} \\
S_{22}= & S_{2} \cap \overline{\operatorname{Pre}\left(S_{1}\right)} \\
= & \left\{\left(y_{1}, y_{2}\right) \in \mathbb{R}^{2} \mid\left(y_{1} \leq-\frac{1}{2} \vee y_{1} \geq \frac{1}{2}\right)\right. \\
& \left.\wedge\left(y_{2} \leq-\frac{1}{2} \vee y_{2} \geq \frac{1}{2}\right)\right\} .
\end{aligned}
$$

At this point the refined partition is given by $\Pi=$ $\left\{S_{1}, S_{21}, S_{22}\right\}$. Choosing now $S_{1}$ and $S_{21}$ from $\Pi$ we compute

$$
\begin{aligned}
\operatorname{Pre}\left(S_{21}\right)= & \left\{\left(y_{1}, y_{2}\right) \in \mathbb{R}^{2} \mid y_{2} \leq-\frac{1}{2} \vee y_{2} \geq \frac{1}{2}\right\} \\
S_{1} \cap \operatorname{Pre}\left(S_{21}\right)= & \left\{\left(y_{1}, y_{2}\right) \in \mathbb{R}^{2} \mid-\frac{1}{2}<y_{1}<\frac{1}{2}\right. \\
& \left.\wedge\left(y_{2} \leq-\frac{1}{2} \vee y_{2} \geq \frac{1}{2}\right)\right\}
\end{aligned}
$$

Again, we verify that $S_{1} \cap \operatorname{Pre}\left(S_{21}\right) \neq \varnothing$ and $S_{1} \cap \operatorname{Pre}\left(S_{21}\right) \neq$ $S_{1}$ which leads to the splitting of $S_{1}$ into $S_{11}$ and $S_{12}$ defined by

$$
\begin{aligned}
S_{11}= & S_{1} \cap \operatorname{Pre}\left(S_{21}\right) \\
= & \left\{\left(y_{1}, y_{2}\right) \in \mathbb{R}^{2} \mid-\frac{1}{2}\right. \\
& \left.<y_{1}<\frac{1}{2} \wedge\left(y_{2} \leq-\frac{1}{2} \vee y_{2} \geq \frac{1}{2}\right)\right\}
\end{aligned}
$$



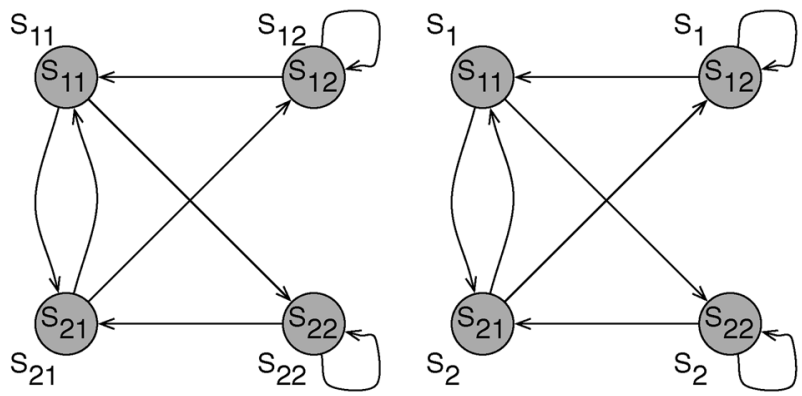

Fig. 3. Finite bisimilar quotient $T_{\Delta}^{\mathcal{P}^{\prime}}$ on the left and $T_{\Delta}^{\mathcal{P}}$ on the right.

$$
\begin{aligned}
S_{12}= & S_{1} \cap \overline{\operatorname{Pre}\left(S_{21}\right)} \\
= & \left\{\left(y_{1}, y_{2}\right) \in \mathbb{R}^{2} \mid-\frac{1}{2}<y_{1}\right. \\
& \left.<\frac{1}{2} \wedge\left(-\frac{1}{2}<y_{2}<\frac{1}{2}\right)\right\} .
\end{aligned}
$$

This splitting leads to the new partition $\Pi=$ $\left\{S_{11}, S_{12}, S_{21}, S_{22}\right\}$ which already defines a bisimulation since

$$
\begin{array}{cc}
S_{11} \cap \operatorname{Pre}\left(S_{11}\right)=\emptyset & S_{12} \cap \operatorname{Pre}\left(S_{11}\right)=S_{12} \\
S_{11} \cap \operatorname{Pre}\left(S_{12}\right)=\emptyset & S_{12} \cap \operatorname{Pre}\left(S_{12}\right)=S_{12} \\
S_{11} \cap \operatorname{Pre}\left(S_{21}\right)=S_{11} & S_{12} \cap \operatorname{Pre}\left(S_{21}\right)=\emptyset \\
S_{11} \cap \operatorname{Pre}\left(S_{22}\right)=S_{11} & S_{12} \cap \operatorname{Pre}\left(S_{22}\right)=\emptyset \\
S_{21} \cap \operatorname{Pre}\left(S_{11}\right)=S_{21} & S_{22} \cap \operatorname{Pre}\left(S_{11}\right)=\emptyset \\
S_{21} \cap \operatorname{Pre}\left(S_{12}\right)=S_{21} & S_{22} \cap \operatorname{Pre}\left(S_{12}\right)=\emptyset \\
S_{21} \cap \operatorname{Pre}\left(S_{21}\right)=\emptyset & S_{22} \cap \operatorname{Pre}\left(S_{21}\right)=S_{21} \\
S_{21} \cap \operatorname{Pre}\left(S_{22}\right)=\emptyset & S_{22} \cap \operatorname{Pre}\left(S_{22}\right)=S_{21} .
\end{array}
$$

This finite bisimulation has four discrete states and is graphically represented in Fig. 3 where initial states are grey colored and observations are represented outside the circles denoting the states.

Example 2.14: Consider now a linear control system described by the following matrices:

$$
A=\left[\begin{array}{ccc}
2 & 0 & -1 \\
-1 & -7 & 11 \\
0 & 4 & 6
\end{array}\right] \quad B=\left[\begin{array}{ll}
1 & 2 \\
1 & 1 \\
1 & 1
\end{array}\right]
$$

It is not difficult to see that controllability holds and the controllability indices are given by $\mu_{1}=2$ and $\mu_{2}=1$. Vector space $V$ is, therefore, spanned by the first column of $B$. Adopting the following matrix representation for the observation map $\Upsilon: \mathbb{R}^{3} \rightarrow$ $\mathbb{R}^{2}$ : it follows that equality $\Upsilon b_{1}=0$ leads to $a_{1}+a_{2}+a_{3}=0$ and $b_{1}+b_{2}+b_{3}=0$. One possible choice for map $\Upsilon$ satisfying these equations is

$$
\Upsilon x=\left[\begin{array}{lll}
0 & 1 & -1 \\
1 & 1 & -2
\end{array}\right]\left[\begin{array}{l}
x_{1} \\
x_{2} \\
x_{3}
\end{array}\right] .
$$

Starting with the following observation space partition:

$$
\begin{aligned}
& S_{1}=\left\{\left(y_{1}, y_{2}\right) \in \mathbb{R}^{2} \mid y_{1}>0 \wedge y_{2}>0\right\} \\
& S_{2}=\left\{\left(y_{1}, y_{2}\right) \in \mathbb{R}^{2} \mid y_{1}>0 \wedge y_{2} \leq 0\right\} \\
& S_{3}=\left\{\left(y_{1}, y_{2}\right) \in \mathbb{R}^{2} \mid y_{1} \leq 0 \wedge y_{2}>0\right\} \\
& S_{4}=\left\{\left(y_{1}, y_{2}\right) \in \mathbb{R}^{2} \mid y_{1} \leq 0 \wedge y_{2} \leq 0\right\}
\end{aligned}
$$

we obtain a finite bisimulation with eight states defined by $T_{\Delta}^{\mathcal{P}}=\left(Q, Q^{0}, \longrightarrow, O, \Upsilon_{\Delta}\right)$ where

$$
\begin{aligned}
Q= & \left\{q_{1}, q_{2}, q_{3}, q_{4}, q_{5}, q_{6}, q_{7}, q_{8}\right\} \quad Q^{0}=Q \\
O= & \left\{S_{1}, S_{2}, S_{3}, S_{4}\right\} \\
\longrightarrow= & \left\{\left(q_{1}, q_{1}\right),\left(q_{1}, q_{2}\right),\left(q_{1}, q_{3}\right),\left(q_{1}, q_{4}\right),\left(q_{3}, q_{1}\right),\left(q_{3}, q_{2}\right)\right\} \\
& \cup\left\{\left(q_{3}, q_{3}\right),\left(q_{3}, q_{4}\right),\left(q_{2}, q_{5}\right),\left(q_{2}, q_{6}\right),\left(q_{2}, q_{7}\right),\left(q_{2}, q_{8}\right)\right\} \\
& \cup\left\{\left(q_{4}, q_{5}\right),\left(q_{4}, q_{6}\right),\left(q_{4}, q_{7}\right),\left(q_{4}, q_{8}\right),\left(q_{5}, q_{1}\right),\left(q_{5}, q_{2}\right)\right\} \\
& \cup\left\{\left(q_{5}, q_{3}\right),\left(q_{5}, q_{4}\right),\left(q_{7}, q_{1}\right),\left(q_{7}, q_{2}\right),\left(q_{7}, q_{3}\right),\left(q_{7}, q_{4}\right)\right\} \\
& \cup\left\{\left(q_{6}, q_{5}\right),\left(q_{6}, q_{6}\right),\left(q_{6}, q_{7}\right),\left(q_{6}, q_{8}\right),\left(q_{8}, q_{5}\right),\left(q_{8}, q_{6}\right)\right\} \\
& \cup\left\{\left(q_{8}, q_{7}\right),\left(q_{8}, q_{8}\right)\right\}
\end{aligned}
$$

$\Upsilon_{\Delta}\left(q_{1}\right)=S_{1} \quad \Upsilon_{\Delta}\left(q_{2}\right)=S_{1} \quad \Upsilon_{\Delta}\left(q_{3}\right)=S_{2} \quad \Upsilon_{\Delta}\left(q_{4}\right)=S_{2}$

$\begin{array}{llll}\Upsilon_{\Delta}\left(q_{5}\right)=S_{3} & \Upsilon_{\Delta}\left(q_{6}\right)=S_{3} & \Upsilon_{\Delta}\left(q_{7}\right)=S_{4} & \Upsilon_{\Delta}\left(q_{8}\right)=S_{4} .\end{array}$

The sets associated with each discrete state were computed using Algorithm 2.10 and are given by

$$
\begin{aligned}
q_{1}: & \left\{\left(x_{1}, x_{2}, x_{3}\right) \in \mathbb{R}^{3} \mid x_{2}-x_{3}>0 \wedge\right. \\
& \left.-x_{1}-11 x_{2}+5 x_{3}>0 \wedge x_{1}+x_{2}-2 x_{3}>0\right\} \\
q_{2}: & \left\{\left(x_{1}, x_{2}, x_{3}\right) \in \mathbb{R}^{3} \mid x_{2}-x_{3}>0 \wedge\right. \\
& \left.-x_{1}-11 x_{2}+5 x_{3} \leq 0 \wedge x_{1}+x_{2}-2 x_{3}>0\right\} \\
q_{3}: & \left\{\left(x_{1}, x_{2}, x_{3}\right) \in \mathbb{R}^{3} \mid x_{2}-x_{3}>0 \wedge\right. \\
& \left.-x_{1}-11 x_{2}+5 x_{3}>0 \wedge x_{1}+x_{2}-2 x_{3} \leq 0\right\} \\
q_{4}: & \left\{\left(x_{1}, x_{2}, x_{3}\right) \in \mathbb{R}^{3} \mid x_{2}-x_{3}>0 \wedge\right. \\
& \left.-x_{1}-11 x_{2}+5 x_{3} \leq 0 \wedge x_{1}+x_{2}-2 x_{3} \leq 0\right\} \\
q_{5}: & \left\{\left(x_{1}, x_{2}, x_{3}\right) \in \mathbb{R}^{3} \mid x_{2}-x_{3} \leq 0\right. \\
& \left.\wedge-x_{1}-11 x_{2}+5 x_{3}>0 \wedge x_{1}+x_{2}-2 x_{3}>0\right\} \\
q_{6}: & \left\{\left(x_{1}, x_{2}, x_{3}\right) \in \mathbb{R}^{3} \mid x_{2}-x_{3} \leq 0\right. \\
& \left.\wedge-x_{1}-11 x_{2}+5 x_{3} \leq 0 \wedge x_{1}+x_{2}-2 x_{3}>0\right\} \\
q_{7}: & \left\{\left(x_{1}, x_{2}, x_{3}\right) \in \mathbb{R}^{3} \mid x_{2}-x_{3} \leq 0 \wedge\right. \\
& \left.-x_{1}-11 x_{2}+5 x_{3}>0 \wedge x_{1}+x_{2}-2 x_{3} \leq 0\right\} \\
q_{8}: & \left\{\left(x_{1}, x_{2}, x_{3}\right) \in \mathbb{R}^{3} \mid x_{2}-x_{3} \leq 0 \wedge\right. \\
& \left.-x_{1}-11 x_{2}+5 x_{3} \leq 0 \wedge x_{1}+x_{2}-2 x_{3} \leq 0\right\} .
\end{aligned}
$$

To illustrate the computation of these sets, we consider $q_{1}$. Starting from partition $\mathcal{P}=\left\{S_{1}, S_{2}, S_{3}, S_{4}\right\}$ 
we obtain the state space partition $\Upsilon^{-1}(\mathcal{P})=$ $\left\{\Upsilon^{-1}\left(S_{1}\right), \Upsilon^{-1}\left(S_{2}\right), \Upsilon^{-1}\left(S_{3}\right), \Upsilon^{-1}\left(S_{4}\right)\right\}$. If $\quad$ we $\quad$ denote by $P$ the set $\Upsilon^{-1}\left(S_{1}\right)$, we have

$$
\begin{aligned}
P= & \left\{x \in \mathbb{R}^{3} \mid x_{2}-x_{3}>0 \wedge x_{1}+x_{2}-2 x_{3}>0\right\} \\
\operatorname{Pre}(P)= & \left\{x \in \mathbb{R}^{3} \mid \exists u \in \mathbb{R}^{2} x_{1}-2 x_{3}-15 x_{2}+u_{2}>0\right. \\
& \left.\wedge-x_{1}-11 x_{2}+5 x_{3}>0\right\} \\
= & \left\{x \in \mathbb{R}^{3} \mid-x_{1}-11 x_{2}+5 x_{3}>0\right\} \\
\operatorname{Pre}(P) \cap P= & \left\{x \in \mathbb{R}^{3} \mid x_{2}-x_{3}>0 \wedge x_{1}+x_{2}-2 x_{3}>0 \wedge\right. \\
& \left.-x_{1}-11 x_{2}+5 x_{3}>0\right\}=q_{1} .
\end{aligned}
$$

\section{LTL FORMULAS AND BÜCHI AUTOMATA}

LTL logic is a very powerful specification mechanism since it allows one to express complex requirements through simple formulas. Even though the use of temporal logic is now widely used for verification of software systems [10], [11], we argue that temporal logic is equally relevant to synthesis problems. In this section, we define LTL syntax and semantics, provide simple examples to illustrate the definitions, and discuss the translation of LTL formulas into Büchi automata.

\section{A. LTL Syntax and Semantics}

We start with a finite set $\mathcal{P}$ of predicates from which more complex formulas can be built. Even though $\mathcal{P}$ can be an arbitrary finite set we shall keep in mind the particular case where $\mathcal{P}$ is the observation space of $T_{\Sigma}^{\mathcal{P}}$. LTL formulas are then obtained through the following recursive definition:

- true, false and $p$ are LTL formulas for all $p \in \mathcal{P}$;

- if $\varphi_{1}$ and $\varphi_{2}$ are LTL formulas, then $\varphi_{1} \wedge \varphi_{2}$ and $\neg \varphi_{1}$ are LTL formulas;

- if $\varphi_{1}$ and $\varphi_{2}$ are LTL formulas, then $\circ \varphi_{1}$ and $\varphi_{1} \mathbf{U} \varphi_{2}$ are LTL formulas.

As usual, disjunction $\varphi_{1} \vee \varphi_{2}$ and implication $\varphi_{1} \Rightarrow \varphi_{2}$ are defined as the abbreviations of $\neg\left(\varphi \wedge \varphi_{2}\right)$ and $\neg \varphi_{1} \vee \varphi_{2}$, respectively. The operator $\circ$ is read as "next," with the meaning that the formula it precedes will be true in the next time step. The second operator $\mathbf{U}$ is read as "until" and the formula $\varphi_{1} \mathbf{U} \varphi_{2}$ specifies that $\varphi_{1}$ must hold until $\varphi_{2}$ holds. From the $\mathbf{U}$ operator, we can define other commonly used operators:

$$
\begin{aligned}
\diamond \varphi & =\operatorname{trueU} \varphi \\
\square \varphi & =\neg \diamond \neg \varphi .
\end{aligned}
$$

Formula $\square \varphi$ is read as "always $\varphi$ " and requires $\varphi$ to be true for all future time, while formula $\diamond \varphi$ reads "eventually $\varphi$ " and states that $\varphi$ will become true at some point in the future. A unique interpretation of LTL formulas is obtained by defining LTL semantics. LTL formulas are interpreted over sequences of predicate values $s \in \mathcal{P}^{\omega}$. Although LTL formulas are usually interpreted over sequences of sets of predicate values, in this paper we identify $\mathcal{P}$ with a finite partition of the observation space and thus for each $t \in \mathbb{N}$ only one predicate is satisfied. We say that string $s$ satisfies formula $\varphi$ at time $t$, denoted by $s(t) \models \varphi$, if formula $\varphi$ holds at time $t$ along trajectory $s$. The satisfaction relation is defined as follows: For any $p \in \mathcal{P}$, LTL formulas $\varphi_{1}, \varphi_{2}$, and $t \in \mathbb{N}$ :

- $s(t) \models p$ iff $p=s(t)$;

- $s(t) \models \neg p$ iff $p \neq s(t)$;

- $s(t) \vDash \varphi_{1} \wedge \varphi_{2}$ iff $s(t) \vDash \varphi_{1}$ and $s(t) \vDash \varphi_{2}$;

- $s(t) \models \circ \varphi_{1}$ iff $s(t+1) \vDash \varphi_{1}$;

- $s(t) \models \varphi_{1} \mathbf{U} \varphi_{2}$ iff $\exists t^{\prime} \geq t$ such that for all $k, t \leq k \leq t^{\prime}$ $s(k) \models \varphi_{1}$ and $s\left(t^{\prime}\right) \models \varphi_{2}$.

Finally, we say that a sequence $s$ satisfies formula $\varphi$ iff $s(0) \models$ $\varphi$.

Example 3.1: As a first example consider the formula $\square p$ for $p \in \mathcal{P}$. This formula defines an invariance property by requiring $p$ to hold for all $t \in \mathbb{N}$. Such specification is useful, for example, in one wants to restrict the activity of a certain control system to a set of operating conditions defined by the predicate $p$. The semantics of $\square p$ can be obtained from the semantics of $\mathbf{U}$ using the definition of $\square$ given in (10) or given directly as $s \models \square \varphi$ iff $s(i) \vDash \varphi$ for all $i \in \mathbb{N}$. It then follows that the unique string $s \in \mathcal{P}^{\omega}$ satisfying $\square p$ is

\section{ррррррррррр $\cdots$}

When each predicate $p \in \mathcal{P}$ is an element of a finite partition of the observation space $O$ of $T_{\Sigma}$, requirement $\square p$ specifies that trajectories of $\Sigma$ should start in the set defined by $p$ and stay in that set forever.

Example 3.2: Consider now the formula $p_{1} \mathbf{U} p_{2}$. According to the previously introduced semantics, every string satisfying this formula is of the form:

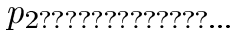

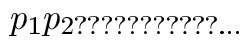

$$
\begin{aligned}
& p_{1} p_{1} p_{2 ? ? ? ? ? ? ? ? ? . .} \\
& p_{1} p_{1} p_{1} p_{2} \text { ???????... } \\
& p_{1} p_{1} p_{1} p_{1} p_{2 ? ? ? ? ? . .}
\end{aligned}
$$

where we have used the symbol? to denote an occurrence of any predicate in $\mathcal{P}$. The first string satisfies $p_{1} \mathbf{U} p_{2}$ by satisfying $p_{2}$, after which formula $p_{1} \mathbf{U} p_{2}$ no longer imposes any constraint on the string. The remaining strings satisfy $p_{1} \mathbf{U} p_{2}$ by initially satisfying $p_{1}$ until they satisfy $p_{2}$ at some later time. Once $p_{2}$ is satisfied, any predicate in $\mathcal{P}$ is allowed to occur in the string since $p_{1} \mathbf{U} p_{2}$ is already true. Operator $\mathbf{U}$ is very useful to capture temporal ordering of control requirements. One can specify, for example, that the temperature and humidity in a building should stay within certain bounds (as specified by predicates on the observation space) until the end of working hours, or that an aircraft should stay at a certain altitude until the descent phase is initiated, etc.

Example 3.3: More complex (and useful) formulas usually involve nesting of temporal operators. One such example is obtained by combining the $\square$ operator with the formula $p_{1} \mathbf{U} p_{2}$ resulting in the formula $p_{1} \mathbf{U} \square p_{2}$. Intuitively this formula requires $p_{2}$ to hold for all time, or that $p_{1}$ holds until at some later 
time $p_{2}$ holds for all future time. A string satisfying $p_{1} \mathbf{U} \square p_{2}$ is necessarily of the form

$$
\begin{aligned}
& p_{2} p_{2} p_{2} p_{2} p_{2} \cdots \\
& p_{1} p_{2} p_{2} p_{2} p_{2} \cdots \\
& p_{1} p_{1} p_{2} p_{2} p_{2} \cdots \\
& p_{1} p_{1} p_{1} p_{2} p_{2} \cdots
\end{aligned}
$$

Formula $p_{1} \mathbf{U} \square p_{2}$ can be used to model convergence towards the operating conditions described by $p_{2}$ through a particular subset of the state-space described by $p_{1}$.

Different combinations of $\square$ and $\mathbf{U}$ result in formulas with different meaning. For example, any string satisfying the formula $\square\left(p_{1} \mathbf{U} p_{2}\right)$ must satisfy $p_{1} \mathbf{U} p_{2}$ at every time step, which implies that at every time step either $p_{2}$ holds or $p_{1}$ holds until $p_{2}$ holds at some future time. Examples of strings satisfying $\square\left(p_{1} \mathbf{U} p_{2}\right)$ are given as follows:

\section{$p_{2} p_{2} p_{2} p_{2} p_{2} p_{2} p_{2} p_{2} p_{2} p_{2} p_{2} p_{2} p_{2} \ldots$ $p_{2} p_{1} p_{2} p_{1} p_{2} p_{1} p_{2} p_{1} p_{2} p_{1} p_{2} p_{1} p_{2} \ldots$ $p_{1} p_{1} p_{1} p_{2} p_{1} p_{1} p_{2} p_{1} p_{1} p_{2} p_{1} p_{1} p_{2} \ldots$ $p_{1} p_{1} p_{1} p_{1} p_{1} p_{1} p_{1} p_{1} p_{1} p_{1} p_{2} p_{2} p_{2} \ldots$}

Example 3.4: We now return to Example 2.13. The only predicate appearing in formula (8), is an element of the observation space of $T_{\Sigma}$. According to LTL semantics and the abbreviation $\diamond_{3} \varphi=\varphi \vee \circ \varphi \vee \circ \circ \varphi \vee \circ \circ \circ \varphi$, some of the infinite strings satisfying (8) are given by

$$
\begin{aligned}
& S_{1} S_{1} S_{1} S_{1} S_{1} S_{1} S_{1} \ldots \\
& S_{1} S_{2} S_{1} S_{2} S_{1} S_{2} S_{1} \ldots \\
& S_{1} S_{1} S_{2} S_{1} S_{1} S_{2} S_{1} \ldots \\
& S_{1} S_{2} S_{2} S_{1} S_{2} S_{2} S_{1} \ldots
\end{aligned}
$$

The reader should verify for himself that in every of the above strings, every length 4 sub-string contains $S_{1}$. This shows that the previous strings satisfy the specification formula since $\mathcal{S} \diamond_{3}$ $S_{1}$ requires that at every time step $t, \diamond_{3} S_{1}$ holds, meaning that $S_{1}$ should hold at $t$ or that it should hold at $t+1, t+2$ or $t+3$. This simple example also shows how tedious and error prone it is to list all the possible strings satisfying the very simple formula (8).

When a LTL formula is interpreted over observed sequences in $L_{\omega}\left(T_{\Sigma}^{\mathcal{P}}\right)$ each predicate corresponds to a subset of $\mathbb{R}^{m}$ and the specification defines how trajectories of $\Sigma$ interact with these sets. This is a convenient and formal way of expressing control requirements for discrete-time linear systems. If every string in $L_{\omega}\left(T_{\Sigma}^{\mathcal{P}}\right)$ satisfies formula $\varphi$ we simply say that $T_{\Sigma}^{\mathcal{P}}$ satisfies $\varphi$ which is denoted by $T_{\Sigma}^{\mathcal{P}} \models \varphi$. We shall use a similar notation for $T_{\Sigma}^{\mathcal{P}^{\prime}}$ even though predicates in $\varphi$ do not correspond to sets in $\mathcal{P}^{\prime}$. We shall use the notation $T_{\Sigma}^{\mathcal{P}^{\prime}} \models \varphi$ when for every $r \in L_{\omega}\left(T_{\Sigma}^{\mathcal{P}^{\prime}}\right), \pi_{\mathcal{P}^{\prime} \mathcal{P}}(r) \models \varphi$. In general it is not the case that $T_{\Sigma}^{\mathcal{P}^{\prime}} \models \varphi$ and a controller $T_{c}$ needs to be constructed to ensure satisfaction of $\varphi$ by the closed-loop system. Such a controller is built from $T_{\Delta}^{\mathcal{P}^{\prime}}$ and a Büchi automaton describing the specification. It is therefore necessary to translate the LTL specification formula $\varphi$ into a Büchi automaton.

\section{B. Büchi Automata}

The strings satisfying a given LTL formula can also be compactly described in terms of a finite operational model. Such model is slightly more complex than a transition system since LTL formulas specify both the finite and infinite behavior of strings. Consider for example the formula $\diamond p=$ true $\mathbf{U} p$ for $p \in \mathcal{P}$. This formula requires $p$ to hold at some time in the future. Given a string $s \in \mathcal{P}^{\omega}$, we cannot decide if $s \models \diamond p$ by looking at a finite prefix of $s$ since $p$ can always appear at a later point in time. This shows that we need to equip transition systems with an additional mechanism describing the behavior of strings "at infinity." These new transition systems are called Büchi automata.

Definition 3.5: A Büchi automaton is a tuple $A=\left(T_{A}, F\right)=$ $\left(\left(Q, Q^{0}, \longrightarrow, O, \Upsilon\right), F\right)$, where $T_{A}$ is a finite transition system and $F \subseteq Q$ is a set of final states. A string $s \in Q^{\omega}$ is a run of $A$ if $s(\overline{1}) \in Q^{0}, s(i) \longrightarrow s(i+1)$ for $i \in \mathbb{N}$ and there exist infinitely many $i \in \mathbb{N}$ such that $s(i) \in F$.

Since every Büchi automaton $A$ carries an underlying transition system structure $T_{A}$, it also defines generated languages and $\omega$-languages. In addition, final states allows one to introduce the notion of accepted language.

Definition 3.6: Let $A=\left(Q, Q^{0}, \longrightarrow, O, \Upsilon, F\right)$ be a Büchi automaton. The language accepted by $A$, denoted by $L_{\omega}(A)$, is defined as

$$
\left\{r \in O^{\omega} \mid r=\Upsilon(s) \text { for some initialized run } s \text { of } A\right\} .
$$

Büchi automata accept languages which are more general than the languages generated by transition systems. Since given a transition system $T$ we can always construct a Büchi automaton $A$ with $F=Q$, leading to $L_{\omega}(A)=L_{\omega}\left(T_{A}\right)=L_{\omega}(T)$, we can regard transition systems as a subclass of Büchi automata. The relevance of Büchi automata comes from the fact that for any LTL formula $\varphi$ it is possible to construct a Büchi automaton $A_{\varphi}$ accepting every string satisfying formula $\varphi$. This fact was first shown by Büchi [60] in the context of decidability of first and second order monadic theories of one successor. Although decidability of the translation between LTL formulas and Büchi automata was settled by Büchi's work, the complexity of such translations has been improved through the years by different authors. The resulting automata are, in the worst case, exponential in the length of the translated formula. However, current practice in computer aided verification shows that such worst case complexity is seldom achieved. Since this translation is well documented in the literature we point the interested reader to the survey [61] and to the algorithms described in [62] and [63] for more details. We now return to the periodic synchronization example converting the specification formula into a Büchi automaton. 


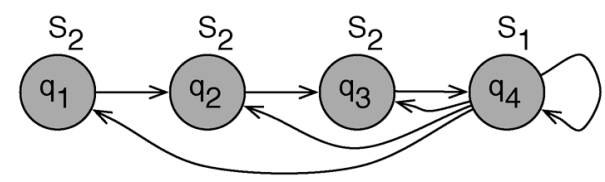

Fig. 4. Transition system $T_{\varphi}$ corresponding to specification formula $\varphi$.

Example 3.7: Recall the specification formula discussed in Example 2.13 that we repeat here for convenience

$$
\varphi=\square \diamond_{3}\left(p_{1} \wedge p_{2}\right)
$$

Specification formula $\varphi$ can be translated into the transition system $T_{\varphi}$ represented in Fig. 4 where $p_{1} \wedge p_{2}$ has been abbreviated by $S_{1}$, and $\neg\left(p_{1} \wedge p_{2}\right)$ by $S_{2}$. In this case, the final states of Büchi automaton $A_{\varphi}$ are all of its states and thus we can equivalently represent $A_{\varphi}$ by its underlying transition system.

Note that starting from any state of $T_{\varphi}$ state $q_{4}$ will be necessarily reached in at most 3 steps. Since the observation associated with $q_{4}$ is $S_{1}$ this implies that at any time step, $S_{1}$ will hold no later than 3 time steps implying that any string generated by $T_{\varphi}$ satisfies $\varphi$.

We shall not discuss further Büchi automata as we will not have the oportunity of using them in this paper. However, they are essential for the construction of a discrete controller or supervisor $T_{c}$ for $T_{\Delta}^{\mathcal{P}^{\prime}}$ enforcing $\varphi$ as discussed in the next section.

\section{SUPERVISORY SYNTHESIS}

The existence of finite bisimulations for linear systems, discussed in Section II, enables the design of controllers enforcing LTL specifications at the purely discrete level. Such control problems on infinite behaviors have been studied in the discrete event systems community [35], [37] and, in this section, we review the results and concepts required for our purposes. We start by introducing a notion of parallel composition between transition systems modeling interaction between components. This interaction can be understood as a form of control where a supervisor is designed to modify (restrict) the behavior of another (transition) system by interconnection.

Definition 4.1: Let $T_{1}=\left(Q_{1}, Q_{1}^{0}, \longrightarrow 1, O, \Upsilon_{1}\right)$ and $T_{2}=$ $\left(Q_{2}, Q_{2}^{0}, \longrightarrow 2, O, \Upsilon_{2}\right)$ be two transition systems with the same observation space $O$. The parallel composition of $T_{1}$ and $T_{2}$ (with observation synchronization) is denoted by

$$
T_{1} \|_{O} T_{2}=\left(Q_{\|}, Q_{\|}^{0}, \longrightarrow{ }_{\|}, O, \Upsilon_{\|}\right)
$$

where

- $Q_{\|}=\left\{\left(q_{1}, q_{2}\right) \in Q_{1} \times Q_{2} \mid \Upsilon_{1}\left(q_{1}\right)=\Upsilon_{2}\left(q_{2}\right)\right\}$;

- $Q_{\|}^{0}=\left\{\left(q_{1}, q_{2}\right) \in Q_{1}^{0} \times Q_{2}^{0} \mid \Upsilon_{1}\left(q_{1}\right)=\Upsilon_{2}\left(q_{2}\right)\right\}$;

- $\left(q_{1}, q_{2}\right) \longrightarrow \|\left(q_{1}^{\prime}, q_{2}^{\prime}\right)$ for $\left(q_{1}, q_{2}\right),\left(q_{1}^{\prime}, q_{2}^{\prime}\right) \in Q_{\|}$iff $q_{1} \longrightarrow 1 q_{1}^{\prime}$ and $q_{2} \longrightarrow 2 q_{2}^{\prime}$;

- $\Upsilon_{\|}\left(q_{1}, q_{2}\right)=\Upsilon_{1}\left(q_{1}\right)=\Upsilon_{2}\left(q_{2}\right)$.

The presented definition of parallel composition is not the usual synchronous product used in the supervisory control literature since we have defined transition systems with observations on the states rather than on the transitions. Nevertheless, the language of transition system $T_{1} \|_{O} T_{2}$ can still be expressed in terms of the languages of $T_{1}$ and $T_{2}$.

Proposition 4.2: Let $T_{1}$ and $T_{2}$ be transition systems with the same observation space $O$. The following equalities are always satisfied:

$$
\begin{aligned}
L\left(T_{1} \|_{O} T_{2}\right) & =L\left(T_{1}\right) \cap L\left(T_{2}\right) \\
L_{\omega}\left(T_{1} \|_{O} T_{2}\right) & =L_{\omega}\left(T_{1}\right) \cap L_{\omega}\left(T_{2}\right) .
\end{aligned}
$$

The previous proposition shows that a controller $T_{c}$ can restrict the behavior of $T_{\Sigma}$ through language intersection in order to eliminate strings $s \in L_{\omega}\left(T_{\Sigma}\right)$ which do not satisfy the specification formula $\varphi$. Furthermore, as asserted in the next result, a controller for $T_{\Sigma}^{\mathcal{P}^{\prime}}$ can be obtained by working with the finite transition system $T_{\Delta}^{\mathcal{P}^{\prime}}$

proposition 4.3: Let $\Sigma$ be a controllable linear system, let $\varphi$ be a LTL formula with predicates denoting sets in a finite partition $\mathcal{P}$ of the observation space of $T_{\Sigma}$ and let $\mathcal{P}^{\prime}$ be the finite refinement of state-space partition $\Upsilon^{-1}(\mathcal{P})$ whose existence is asserted by Theorem 2.8. There exists a controller $T_{c}$ satisfying $T_{c} \|_{O} T_{\Sigma}^{\mathcal{P}^{\prime}} \models \varphi$ iff there exists a controller $T_{c}$ satisfying $T_{c} \|_{O}$ $T_{\Delta}^{\mathcal{P}^{\prime}} \models \varphi$.

Proof: It follows at once from the properties of bisimulation, see for example [53], that for any transition system $T_{c}, T_{\Sigma}^{\mathcal{P}^{\prime}} \cong T_{\Delta}^{\mathcal{P}^{\prime}}$ implies $T_{c}\left\|_{O} T_{\Sigma}^{\mathcal{P}^{\prime}} \cong T_{c}\right\|_{O} T_{\Delta}^{\mathcal{P}^{\prime}}$. The result now follows from Proposition 2.4.

At this point the reader may be wondering why the previous result is concerned with the existence of a controller for $T_{\Sigma}^{\mathcal{P}^{\prime}}$ and not for $T_{\Sigma}^{\mathcal{P}}$. Since a controller modifies the behavior of the system to be controlled by parallel composition with observation synchronization, working with $T_{\Sigma}^{\mathcal{P}^{\prime}}$ is preferable as the observation space of $T_{\Sigma}^{\mathcal{P}^{\prime}}$ offers more detailed information regarding the dynamics of $T_{\Sigma}$ than the observation space of $T_{\Sigma}^{\mathcal{P}}$.

Recalling that for any LTL formula $\varphi$ we can always construct a Büchi automaton $A_{\varphi}$ recognizing every string satisfying $\varphi$ and recalling that from $T_{\Delta}^{\mathcal{P}^{\prime}}$ we can construct a Büchi automaton $A_{\Delta}^{\mathcal{P}^{\prime}}$ satisfying $L_{\omega}\left(\dot{A}_{\Delta}^{\mathcal{P}^{\prime}}\right)=L_{\omega}\left(T_{\Delta}^{\mathcal{P}^{\prime}}\right)$, the problem of constructing a controller enforcing $\varphi$ can be conceptually reduced to the following steps.

1) Construct $T_{\Sigma}^{\mathcal{P}^{\prime}}$ from $\Sigma$ and $\mathcal{P}$.

2) Construct $T_{\Delta}^{\mathcal{P}^{\prime}}$ from $T_{\Sigma}^{\mathcal{P}^{\prime}}$.

3) Construct $A_{\Delta}^{\mathcal{P}^{\prime}}$ from $T_{\Delta}^{\mathcal{P}^{\prime}}$.

4) Construct $A_{\varphi}$ from $\varphi$.

5) Construct a Büchi automaton controller $A_{c}$ satisfying $\pi_{\mathcal{P}^{\prime} \mathcal{P}}\left(L_{\omega}\left(A_{c}\right) \cap L_{\omega}\left(A_{\Delta}^{\mathcal{P}^{\prime}}\right)\right) \subseteq L_{\omega}\left(A_{\varphi}\right)$.

The first four steps have already been described in this paper and the fifth step has been extensively studied in the discrete-event systems literature [36], [64]-[66]. For the purposes of this paper we will simply assume the existence of $A_{c}$ defined in (5). Note that if no such $A_{c}$ exists, then Proposition 4.3 asserts that no controller for $T_{\Sigma}^{\mathcal{P}^{\prime}}$ (and consequently for $T_{\Sigma}^{\mathcal{P}}$ ) exists. Furthermore, we will also assume that $A_{c}$ can be modeled by a transition system, that is, there exists a transition system $T_{c}$ satisfying $L_{\omega}\left(T_{c}\right) \cap L_{\omega}\left(A_{\Delta}^{\mathcal{P}^{\prime}}\right)=L_{\omega}\left(A_{c}\right) \cap L_{\omega}\left(A_{\Delta}^{\mathcal{P}^{\prime}}\right)$. As discussed in [36], a finite supervisor that is implementable (in software, hardware or software and hardware) necessarily has finite memory and therefore can only restrict the infinite behavior of 


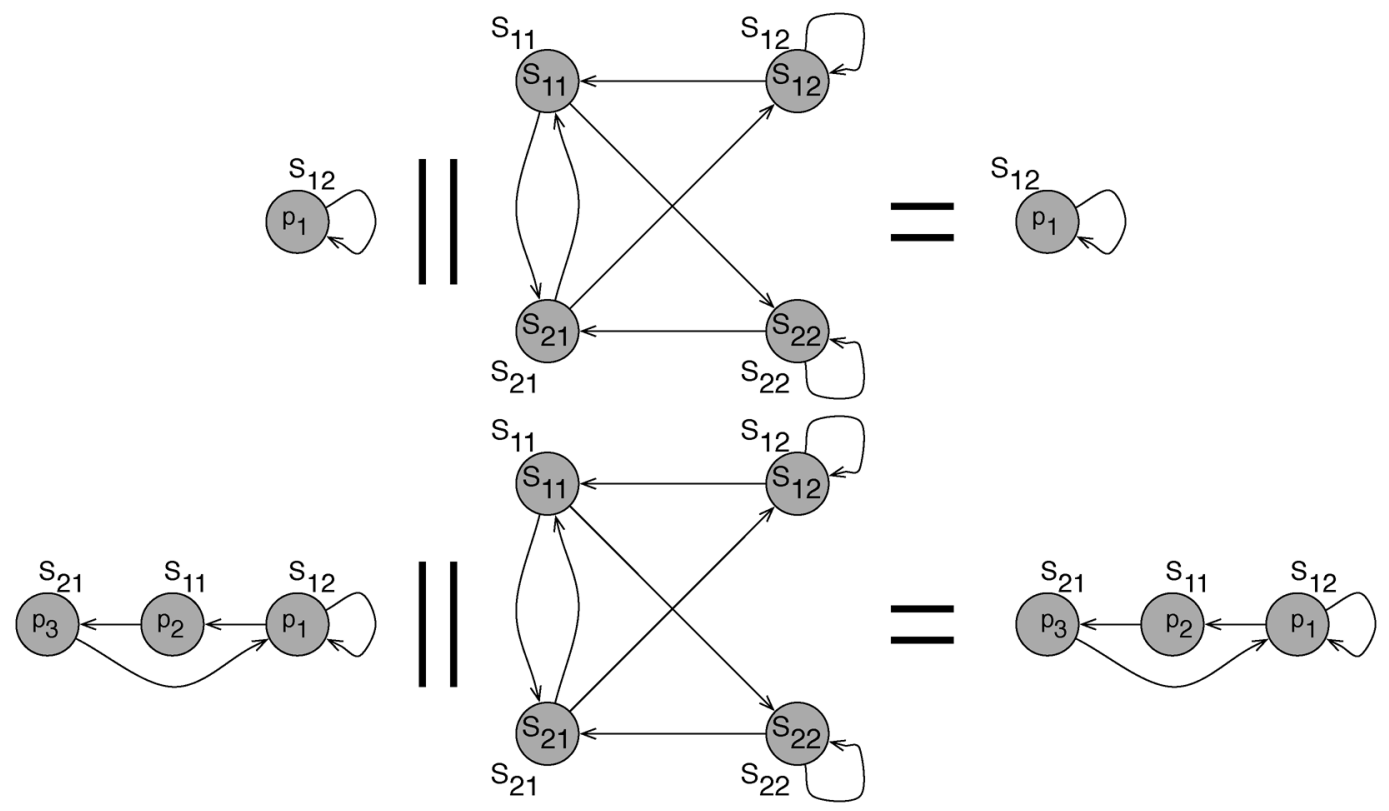

Fig. 5. Parallel composition of $T_{\Delta}^{\mathcal{P}^{\prime}}$ with two different controllers for the problem described in Example 2.13.

$A_{\Delta}^{\mathcal{P}^{\prime}}$ based on finite length observations. Therefore, for any implementable Büchi automaton controller $A_{c}$ enforcing $\varphi$ there exists a finite transition system $T_{c}$ satisfying $L_{\omega}\left(T_{c} \| T_{\Delta}^{\mathcal{P}^{\prime}}\right)=$ $L_{\omega}\left(T_{c}\right) \cap L_{\omega}\left(T_{\Delta}^{\mathcal{P}^{\prime}}\right)=L_{\omega}\left(A_{c}\right) \cap L_{\omega}\left(A_{\Delta}^{\mathcal{P}^{\prime}}\right)$. We refer the reader to [36], [64]-[66] for more details on the existence and computation of $T_{c}$ and return to Example 2.13.

Example 4.4: Fig. 5 shows two different controllers enforcing LTL formula (8) on the transition system displayed in Fig. 3. We can see that both controllers enforce LTL formula (8) on $T_{\Delta}$ since on the first case the language of the parallel composition consists of strings of the form $S_{1} S_{1} S_{1} S_{1} \ldots$, while in the second case it consists of strings in which occurrence of $S_{2}$ (if any) is immediately followed by an occurrence of $S_{1}$.

\section{REFINING THE ClOSED-LoOP}

In the previous section, we outlined how a finite controller $T_{c}$ for $T_{\Delta}^{\mathcal{P}^{\prime}}$ enforcing a desired LTL specification can be obtained. In this section, we will see that we can also extract from $T_{c}$ the continuous inputs required to enforce the specification on $\Sigma$. The explicit modeling of the control inputs available to $\Sigma$ will result in a hybrid closed-loop behavior. This motivates the introduction of discrete-time linear hybrid systems and their corresponding transition systems.

Definition 5.1: A discrete-time linear hybrid system $H=$ $\left(X, X^{0},\left\{A_{q}, B_{q}\right\}_{q \in Q}, \delta, U\right)$ consists of the following elements.

- The state-space $X=\coprod_{q \in Q} \mathbb{R}^{n_{q}}$ where $Q$ is a finite set of states and $n_{q} \in \mathbb{N}$ for each $q \in Q$.

- A set of initial states $X^{0} \subseteq X$.

- The continuous dynamics $\left\{A_{q}, B_{q}\right\}_{q \in Q}$ where for each $q \in Q,\left(A_{q}, B_{q}\right) \in \mathbb{R}^{n_{q} \times n_{q}} \times \mathbb{R}^{n_{q} \times m_{q}}$ defines a discrete-time linear control system $x(t+1)=A_{q} x(t)+$ $B_{q} u(t)$ with inputs restricted to the set $U(q(t), x(t)) \subseteq$ $\mathbb{R}^{m_{q}}$.
- The discrete dynamics $\delta: Q \times \mathbb{R}^{n_{q}} \rightarrow 2^{Q}$ which assigns to each discrete $q \in Q$ and continuous $x \in \mathbb{R}^{n_{q}}$ state the discrete successor states $\delta(q(t), x(t)) \subseteq Q$.

Similarly to the purely continuous case, hybrid systems can also be embedded into the class of transition systems. Assuming the continuous dynamics to be controllable we can define transition system

$$
T_{H}=\left(X, X^{0}, \longrightarrow_{H}, O, \Upsilon\right)
$$

associated with a discrete-time linear hybrid system $H=\left(X, X^{0},\left\{A_{q}, B_{q}\right\}_{q \in Q}, \delta, U\right)$ by $(q, x) \longrightarrow_{H}\left(q^{\prime}, x^{\prime}\right)$ iff $x^{\prime}=A_{q} x+B_{q} u, q^{\prime} \in \delta(q, x)$ and $u \in U(q, x)$. The observation set and map are defined by $O=\coprod_{q \in Q} O_{q}$ and $\Upsilon(q, x)=\Upsilon_{q}(x)$ where $O_{q}$ and $\Upsilon_{q}$ are the observation set and map associated with the control systems defined by $\left(A_{q}, B_{q}\right)$ for each $q \in Q$. The importance of embedding linear hybrid systems into the class of transition systems resides in the possibility of formally defining a notion of correct implementation.

Definition 5.2: Let $\Sigma$ be a controllable linear system, let $\varphi$ be a LTL formula with predicates denoting sets in a finite partition $\mathcal{P}$ of the observation space of $T_{\Sigma}$ and let $T_{c}$ be a controller enforcing $\varphi$ on the finite bisimilar quotient $T_{\Delta}^{\mathcal{P}^{\prime}}$, that is, $T_{c} \|_{O} T_{\Sigma}^{\mathcal{P}^{\prime}} \models \varphi$. Linear hybrid system $H$ is said to be a correct implementation of the closed-loop behavior $T_{c} \|_{O} T_{\Sigma}^{\mathcal{P}^{\prime}}$ if $T_{c} \|_{O} T_{\Sigma}^{\mathcal{P}^{\prime}} \cong T_{H}$.

A hybrid implementation $H=\left(X, X^{0},\left\{A_{q}, B_{q}\right\}_{q \in Q}, \delta, U\right)$ of a desired closed-loop behavior $T_{c} \|_{O} T_{\Sigma}^{\mathcal{P}^{\prime}}$ can be immediately obtained from $T_{c} \|_{O} T_{\Sigma}^{\mathcal{P}^{\prime}}=\left(Q_{\|}, Q_{\|}^{0}, \longrightarrow \|, O, \Upsilon_{\|}\right)$by defining

$$
\begin{aligned}
X & =Q_{\|} \\
X^{0} & =Q_{\|}^{0} \\
A_{q} & =A
\end{aligned}
$$




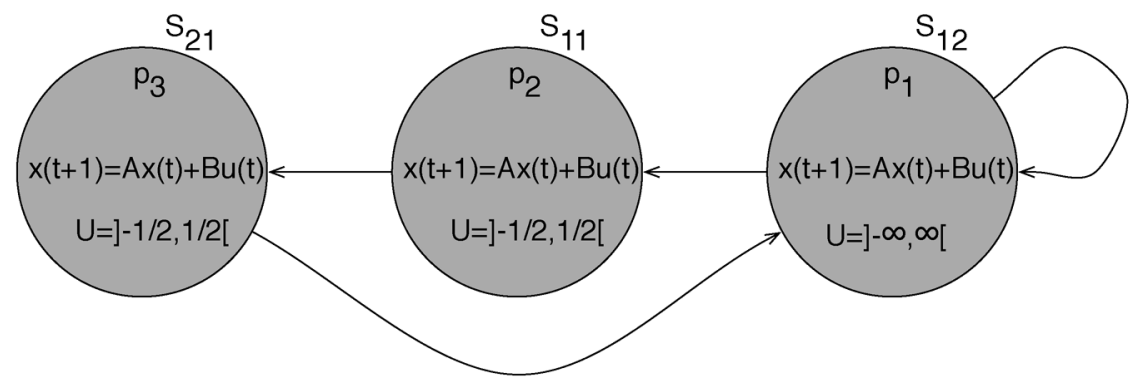

Fig. 6. Hybrid implementation of the closed-loop behavior enforced by the second supervisor represented in Fig. 5 .

$$
\begin{aligned}
B_{q} & =B \\
\delta(q, x) & =\left\{q^{\prime} \in Q \mid q \longrightarrow \| q^{\prime}\right\} \\
U(q, x) & =\left\{u \in \mathbb{R}^{m} \mid \pi_{\mathcal{P}^{\prime}}(A x+B u) \in \Upsilon_{\|}(\delta(q, x))\right\}
\end{aligned}
$$

The construction of $H$ represents the last step required for the solution of Problem 1.1 as we now summarize in the following result.

Theorem 5.3: Let $\Sigma$ be a controllable linear system satisfying Assumptions A.I) and A.II) and defined by matrices $A$ and $B$ with rational entries. For any LTL formula $\varphi$ with predicates denoting semi-linear sets on the observation space of $T_{\Sigma}$, it is decidable to determine the existence of a controller $T_{c}$ satisfying $T_{c} \|_{O} T_{\Sigma}^{\mathcal{P}^{\prime}} \models \varphi$. Furthermore, when such a controller exists it admits the hybrid implementation defined by (11) through (16) which is effectively computable.

Proof: By Corollary 2.12, we can effectively compute $T_{\Delta}^{\mathcal{P}^{\prime}}$. Since we can also effectively compute $A_{\varphi}$ from $\varphi$, it follows from standard results in supervisory control [36], [64]-[66] that it is decidable to determine the existence of a controller $T_{c}$ for $T_{\Delta}^{\mathcal{P}^{\prime}}$ and also that $T_{c}$ is effectively computable. The result now follows from the fact that steps (11) through (16) are effectively computable since the sets denoted by $\varphi$ are semi-linear.

It is important to emphasize that the resulting hybrid controller implicitly defined by $H$ can be obtained in a totally automated fashion. The closed-loop system is still a control system in the sense that at every state different future evolutions are possible under the action of different input values. This is natural since the closed-loop model can now be further controlled to satisfy additional objectives or optimized to extremize certain performance criteria. Another important characteristic of the presented method is the automatic synthesis of both the switching logic (implemented by software) and the continuous aspects of control. In fact, a software implementation of the controller implicitly defined by $H$ can be automatically generated from $H$ by translating each discrete state of $H$ into code reading the state $x$ from sensors, computing $u$ based on $q$ and $x$ and sending $u$ to the actuators. This fact is especially important since verification of hybrid systems is currently limited to systems with very simple continuous dynamics such as timed automata. The proposed approach thus results in systems that satisfy the specification by design while enlarging the class of system that can be shown to operate correctly.

Example 5.4: We now illustrate the construction of the hybrid implementation of the closed-loop systems displayed in Fig. 5. We focus on the construction of $U$ which is the only nontrivial

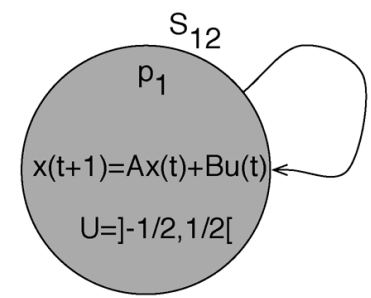

Fig. 7. Hybrid implementation of the closed-loop behavior enforced by the first supervisor represented in Fig. 5.

element in the definition of $H$. The first closed-loop system in Fig. 5 consists of a single discrete state and the corresponding set $U$ is defined by

$$
\begin{gathered}
U\left(p_{1}, x\right)=\left\{u \in \mathbb{R} \mid \pi_{\mathcal{P}^{\prime}}(A x+B u) \in\left\{p_{1}\right\}\right\} \\
=\left\{u \in \mathbb{R} \mid-\frac{1}{2}<u<\frac{1}{2}\right\} .
\end{gathered}
$$

The resulting hybrid implementation is represented in Fig. 7.

The second supervisor has three discrete states for which we need to compute the input set $U$. For discrete state $p_{1}$, we have

$$
\begin{aligned}
U\left(p_{1}, x\right) & =\left\{u \in \mathbb{R} \mid \pi_{\mathcal{P}^{\prime}}(A x+B u) \in\left\{p_{1}, p_{2}\right\}\right\} \\
& =\left\{u \in \mathbb{R} \mid-\frac{1}{2}<u<\frac{1}{2} \vee\left(u \leq-\frac{1}{2} \vee u \geq \frac{1}{2}\right)\right\} \\
& =\mathbb{R}
\end{aligned}
$$

while for states $p_{2}$ and $p_{3}$ the set $U$ is given by:

$$
\begin{aligned}
& U\left(p_{2}, x\right)=\left\{u \in \mathbb{R} \mid-\frac{1}{2}<u<\frac{1}{2}\right\} \\
& U\left(p_{3}, x\right)=\left\{u \in \mathbb{R} \mid-\frac{1}{2}<u<\frac{1}{2}\right\}
\end{aligned}
$$

The resulting hybrid implementation is represented in Fig. 6.

\section{DISCUSSION}

This paper presented an approach for the fully automated synthesis of controllers enforcing LTL specifications for linear systems. The resulting controllers are of hybrid nature combining the continuous dynamics of the original control system with the switching logic required to implement the desired specification. We can thus see these hybrid models as abstract descriptions of the embedded software required for its implementation. Since the resulting closed-loop system is guaranteed to satisfy the specification by construction, the presented synthesis technique enlarges the class of embedded systems for which formal guarantees of operation can be given. 
The proposed approach can be further improved in terms of complexity. Compositional design techniques where different controllers are designed for different aspects of the specification and later combined into a controller for the overall specification allow one to overcome the complexity of translating LTL formulas into Büchi automata. Similarly, design with coarser finite abstractions of $T_{\Sigma}^{\mathcal{P}^{\prime}}$ than $T_{\Delta}^{\mathcal{P}^{\prime}}$ sidesteps the complexity involved in the construction of $T_{\Delta}^{\mathcal{P}^{\prime}}$. The authors are currently investigating these issues as well as synthesis for other temporal logics such as CTL and $\mu$-calculus.

\section{REFERENCES}

[1] B. Dutertre and V. Stavridou, "Formal requirements analysis of an avionics control system," IEEE Trans. Software Eng., vol. 23, no. 5, pp. 267-278, Oct. 1997.

[2] M. Lansdaal and L. Lewis, "Boeing's 777 systems integration lab," IEEE Instrum. Meas. Mag., vol. 3, no. 3, pp. 13-18, Mar. 2000.

[3] J. Teutsch and E. Hoffman, "Aircraft in the future ATM system-Exploiting the 4D aircraft trajectory," in Proc. 23rd Digital Avionics Syst. Conf., 2004, vol. 1, pp. 3.B.2.1-3.B.2.22.

[4] J. Cook, S. Jing, and J. Grizzle, "Opportunities in automotive powertrain control applications," in Proc. 2002 Int. Conf. Control Appl., 2002, pp. xlii-li.

[5] Z. Sun and K. Hebbale, "Challenges and opportunities in automotive transmission control," in Proc. Amer. Control Conf., 2005, pp. 3284-3289.

[6] J. Khurshid and H. Bing-Rong, "Military robots-A glimpse from today and tomorrow," in Proc. 8th Control, Automation, Robotics Vision Conf., 2004, pp. 771-777.

[7] C. Morris, S. Stauth, and B. Parviz, "Self-assembly for microscale and nanoscale packaging: Steps toward self-packaging," IEEE Trans. Adv. Packag., vol. 28, no. 4, pp. 600-611, Nov. 2005.

[8] P. Dario, M. Carrozza, E. Guglielmelli, C. Laschi, A. Menciassi, S. Micera, and F. Vecchi, "Robotics as a future and emerging technology: Biomimetics, cybernetics, and neuro-robotics in european project," IEEE Robot. Automat. Mag., vol. 12, no. 2, pp. 29-45, Feb. 2005.

[9] T. A. Henzinger, P. W. Kopke, A. Puri, and P. Varaiya, "What's decidable about hybrid automata?," J. Comput. Syst. Sci., vol. 57, pp. 94-124, 1998.

[10] Z. Manna and A. Pnueli, The Temporal Logic of Reactive and Concurrent Systems: Specification. Berlin, Germany: Springer-Verlag, 1992.

[11] E. M. M. Clarke, D. Peled, and O. Grumberg, Model Checking. Cambridge, MA: MIT Press, 1999.

[12] K. L. McMillan, Symbolic Model Checking. Norwell, MA: Kluwer, 1993.

[13] S. Tripakis, "Undecidable problems in decentralized observation and control for regular languages," Inform. Process. Lett., vol. 90, no. 1, pp. 21-28, 2004.

[14] R. Alur and D. Dill, "A theory of timed automata," Theoret. Comput. Sci., vol. 126, pp. 183-235, 1994.

[15] R. Alur, C. Courcoubetis, N. Halbwachs, T. Henzinger, P. Ho, X. Nicollin, A. Olivero, J. Sifakis, and S. Yovine, "Hybrid automata: An algorithmic approach to specification and verification of hybrid systems," Theoret. Comput. Sci., vol. 138, pp. 3-34, 1995.

[16] A. Puri and P. Varaiya, "Decidability of hybrid systems with rectangular inclusions," Comput. Aided Verif., pp. 95-104, 1994.

[17] T. Henzinger and R. Majumdar, "Symbolic model checking for rectangular hybrid systems," in TACAS 2000: Tools and Algorithms for the Construction and Analysis of Systems, ser. Lecture Notes in Computer Science, S. Graf, Ed. New York: Springer-Verlag, 2000.

[18] G. Lafferriere, G. J. Pappas, and S. Sastry, "O-minimal hybrid systems," Math. Control, Signals Syst., vol. 13, no. 1, pp. 1-21, Mar. 2000.

[19] R. Alur, T. A. Henzinger, G. Lafferriere, and G. J. Pappas, "Discrete abstractions of hybrid systems," Proc. IEEE, vol. 88, no. 7, pp. 971-984, Jul. 2000

[20] M. Broucke, "A geometric approach to bisimulation and verification of hybrid systems," in Hybrid Systems: Computation and Control, ser. Lecture Notes in Computer Science, F. W. Vaandrager and J. H. van Schuppen, Eds. New York: Springer-Verlag, 1999, vol. 1569, pp. 61-75.

[21] J. Stiver, X. Koutsoukos, and P. Antsaklis, "An invariant based approach to the design of hybrid control systems," Int. J. Robust Nonlinear Control, vol. 11, no. 5, pp. 453-478, 2001.
[22] E. Asarin, G. Schneider, and S. Yovine, "On the decidability of the reachability problem for planar differential inclusions," in Hybrid Systems: Computation and Control, ser. Lecture Notes in Computer Science, M. D. D. Benedetto and A. Sangiovanni-Vincentelli, Eds. New York: Springer-Verlag, 2001, vol. 2034, pp. 89-104.

[23] A. Chotinan and B. Krogh, "Verification of infinite state dynamical systems using approximate quotient transition systems," IEEE Trans. Autom. Control, vol. 46, no. 9, pp. 1401-1410, Sep. 2001.

[24] J. Cury, B. Krogh, and T. Ninomi, "Synthesis of supervisory controllers for hybrid systems based on approximating automata," IEEE Trans. Autom. Control, vol. 43, no. 4, pp. 564-568, Apr. 1998.

[25] A. Tiwari and G. Khanna, "Series of abstractions for hybrid automata," in Hybrid Systems: Computation and Control, ser. Lecture Notes in Computer Science, C. Tomlin and M. R. Greenstreet, Eds. New York: Springer-Verlag, 2002, vol. 2289, pp. 465-478.

[26] A. Bicchi, A. Marigo, and B. Piccoli, "On the reachability of quantized control systems," IEEE Trans. Autom. Control, vol. 47, no. 4, pp. 546-563, Apr. 2002.

[27] S. Pancanti, L. Leonardi, L. Pallottino, and A. Bicchi, "Optimal control of quantized linear systems," in Hybrid Systems: Computation and Control, ser. Lecture Notes in Computer Sience, C. Tomlin and M. R. Greenstreet, Eds. New York: Springer-Verlag, 2002, pp. 351-363.

[28] Y. Chitour and B. Piccoli, "Controllability for discrete systems with a finite control set," Math. Control, Signals Syst., vol. 14, no. 2, pp. 173-193, 2001.

[29] N. Elia and S. K. Mitter, "Stabilization of linear systems with limited information," IEEE Trans. Autom. Control, vol. 46, no. 9, pp. 1384-1400, Sep. 2001.

[30] R. W. Brockett and D. Liberzon, "Quantized feedback stabilization of linear systems," IEEE Trans. Autom. Control, vol. 45, no. 7, pp. 1279-1289, Jul. 2000.

[31] M. Antoniotti and B. Mishra, "Discrete-event models + temporal logic = supervisory controller: Automatic synthesis of locomotion controllers," in Proc. IEEE Int. Conf. Robotics Automation, 1995, pp. 1441-1446.

[32] T. Moor and J. M. Davoren, "Robust controller synthesis for hybrid systems using modal logic," in Hybrid Systems: Computation and Control, ser. Lecture Notes in Computer Science, M. D. D. Benedetto and A. Sangiovanni-Vincentelli, Eds. New York: Springer-Verlag, 2001, vol. 2034.

[33] L. Habets and J. H. van Schuppen, "Control of piecewise-linear hybrid systems on simplices and rectangles," in Hybrid Systems: Computation and Control, ser. Lecture Notes in Computer Sience, M. D. D. Benedetto and A. Sangiovanni-Vincentelli, Eds. New York: Springer-Verlag, 2001, vol. 2034, pp. 261-274.

[34] R. Vidal, S. Schaffert, O. Shakernia, J. Lygeros, and S. Sastry, "Decidable and semi-decidable controller synthesis for classes of discrete time hybrid systems," in Proc. 40th IEEE Conf. Decision Control, Orlando, FL, Dec. 2001, pp. 1243-1248.

[35] P. J. Ramadage and W. M. Wonham, "The control of discrete event systems," Proc. IEEE, vol. 77, no. 1, pp. 81-98, Jan. 1989.

[36] R. Kumar and V. Garg, Modeling and Control of Logical Discrete Event Systems. Norwell, MA: Kluwer, 1995.

[37] C. Cassandras and S. Lafortune, Introduction to Discrete Event Systems. Norwell, MA: Kluwer, 1999.

[38] S. Jiang and R. Kumar, "Supervisory control of discrete event systems with CTL* temporal logic specifications," in Proc. 40th IEEE Conf. Decision Control, Orlando, FL, Dec. 2001, vol. 5, pp. 4122-4127.

[39] J. S. Ostroff, Temporal Logic for Real-Time Systems. New York: Wiley, 1989.

[40] E. A. Emerson and E. M. Clarke, "Using branching time temporal logic to synthesize synchronization skeletons," Sci. Comput. Program., vol. 2, pp. 241-266, 1982.

[41] Z. Manna and P. Wolper, "Synthesis of communication processes from temporal logic specifications," ACM Trans. Program. Lang. Syst., vol. 6, pp. 68-93, 1984.

[42] P. Madhusudan and P. Thiagarajan, "Branching time controllers for discrete event systems," Theoret. Comput. Sci., vol. 274, pp. 117-149, Mar. 2002.

[43] — "A decidable class of asynchronous distributed controllers," in Proc. 13th Int. Conf. Concurrency Theory, L. Brim, M. Kret'ýnsk'y, and A. Kucera, Eds., 2002, vol. 2421, pp. 145-160.

[44] I. Castellani, M. Mukund, and P. Thiagarajan, "Synthesizing distributed transition systems from global specifications," in Proc. Foundations Software Technology Theoretical Computer Science, 1999, vol. 1739, pp. 219-231. 
[45] L. de Alfaro, T. A. Henzinger, and F. Y. C. Mang, "The control of synchronous systems," in 11th Int. Conf. Concurrency Theory, C. Palamidessi, Ed., 2000, vol. 1877, pp. 458-473.

[46] _ - "The control of synchronous systems, Part ii," in Proc. 12th Int. Conf. Concurrency Theory, K. G. Larsen and M. Nielsen, Eds., 2001, vol. 2154 , pp. $566-582$.

[47] M. Faella, S. L. Torre, and A. Murano, "Dense real-time games," in Proc. 17th Annu. IEEE Symp. Logic Comput. Sci., Copenhagen, Denmark, Jul. 2002, pp. 167-176.

[48] Y. Abdeddaim and O. Maler, "Preemptive job-shop scheduling using stopwatch automata," in Proc. Tools Algorithms Construction Analysis Syst., 2002, vol. 2280, pp. 113-126.

[49] G. Behrmann, A. Fehnker, T. Hune, K. G. Larsen, P. Pettersson, J. Romijn, and F. W. Vaandrager, "Minimum-cost reachability for priced timed automata," in Hybrid Systems: Computation and Control, ser. Lecture Notes in Computer Science, M. D. D. Benedetto and A. Sangiovanni-Vincentelli, Eds. New York: Springer-Verlag, 2001, vol. 2034 , pp. $147-161$.

[50] R. Alur, S. L. Torre, and G. Pappas, "Optimal paths in weighted timed automata," in Hybrid Systems: Computation and Control, ser. Lecture Notes in Computer Science, M. D. D. Benedetto and A. SangiovanniVincentelli, Eds. New York: Springer-Verlag, 2001, vol. 2034, pp. 49-62.

[51] D. D. Souza and P. Madhusudan, "Timed control synthesis for external specifications," in Proc. 19th Annu. Symp. Theoretical Aspects Computer Science, H. Alt and A. Ferreira, Eds., 2002, vol. 2285, pp. 571-582.

[52] J. E. Hopcroft and J. D. Ullman, Introduction to Automata Theory, Languages and Computation. Reading, MA: Addison-Wesley, 1979.

[53] R. Milner, Communication and Concurrency. Upper Saddle River, NJ: Prentice-Hall, 1989.

[54] M. Browne, E. Clarke, and O. Grumberg, "Characterizing finite Kripke structures in propositional temporal logic," Theoret. Comput. Sci., vol. 59, pp. 115-131, 1988.

[55] P. Brunovsky, "A classification of linear controllable systems," Kybernetika, vol. 6, no. 3, pp. 173-188, 1970 .

[56] R. E. Kalman, "Kronecker invariants and feedback," in Ordinary Differential Equations, L. Weiss, Ed. New York: Academic, 1972, pp. 459-471.

[57] A. Bouajjani, J. Fernandez, and N. Halbwachs, "Minimal model generation," in CAV90: Computer Aided Verification, ser. Lecture Notes in Computer Science, R. Kurshan and E. M. Clarke, Eds. New York: Springer-Verlag, 1990, vol. 531, pp. 197-203.

[58] P. Kanellakis and S. Smolka, "CCS expressions, finite-state processes, and three problems of equivalence," Inform. Comput., vol. 86, pp. 43-68, 1990.

[59] J. Bochnak, M. Coste, and M.-F. Roy, Real Algebraic Geometry. New York: Springer-Verlag, 1998.

[60] J. R. Buchi, "On a decision method in restricted second order arithmetic," in Proc. Internat. Congr. Logic, Method and Philos. Sci., Stanford, CA, 1962, pp. 1-12, Stanford Univ. Press.

[61] P. Wolper, "Constructing automata from temporal logic formulas: A tutorial," in Lectures on Formal Methods and Performance Analysis, ser. Lecture Notes in Computer Science, E. Brinksma, H. Hermanns, and J. P. Katoen, Eds. New York: Springer-Verlag, 2000, vol. 2090.

[62] F. Somenzi and R. Bloem, "Efficient buchi automata from ltl formulae," in Proc. 12th Int. Conf. Computer Aided Verification, E. A. Emerson and A. P. Sistla, Eds., 2000, vol. 1855, pp. 248-263.

[63] R. Gerth, D. Peled, M. Y. Vardi, and P. Wolper, "Simple on-the-fly automatic verification of linear temporal logic," in Proc. 15th IFIP Workshop Protocol Specification, Testing and Verification, P. Dembinski and M. Sredniawa, Eds., Warsaw, Poland, Jun. 1996, pp. 3-18.

[64] P. Ramadge, "Some tractable supervisory control problems for discrete event systems modeled by Buchi automata," IEEE Trans. Autom. Control, vol. 34, no. 1, pp. 10-19, Jan. 1989.
[65] R. Kumar, V. Garg, and S. Markus, "On supervisory control of sequential behaviors," IEEE Trans. Autom. Control, vol. 37, no. 12, pp. 1978-1985, Dec. 1992

[66] J. Thistle and W. M. Wonham, "Supervision of infinite behavior of discrete-event systems," SIAM J. Control Optim., vol. 32, no. 4, pp. 1098-1113, Jul. 1994.

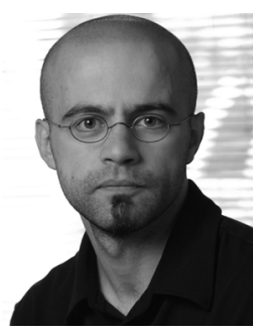

Paulo Tabuada (M'00-M'02) was born in Lisbon, Portugal, one year after the Carnation Revolution. He received the "Licenciatura" degree in aerospace engineering from the Instituto Superior Técnico, Lisbon, Portugal, in 1998, and the Ph.D. degree in electrical and computer engineering from the Institute for Systems and Robotics, a private research institute associated with Instituto Superior Técnico, in 2002.

Between January 2002 and July 2003, he was a Postdoctoral Researcher at the University of Pennsylvania, Philadelphia. He was an Assistant Professor in the Department of Electrical Engineering, the University of Notre Dame, Notre Dame, IN. Currently, he is with the Electrical Engineering Department, the University of California, Los Angeles. His research interests include modeling, analysis and control of real-time, embedded, networked and distributed systems; geometric control theory and mathematical systems theory.

Dr. Tabuada was the recipient of the Francisco de Holanda Prize in 1998 for the best research project with an artistic or aesthetic component awarded by the Portuguese Science Foundation. He was a finalist for the Best Student Paper Award at both the 2001 American Control Conference and the 2001 IEEE Conference on Decision and Control and he was the recipient of a National Science Foundation CAREER award in 2005. He coedited the volume Networked Embedded Sensing and Control published in Springer's Lecture Notes in Control and Information Sciences series.

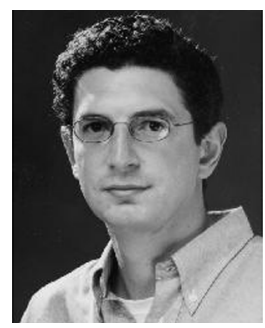

George J. Pappas (S'91-M'98-SM'04) received the $\mathrm{Ph} . \mathrm{D}$. degree in electrical engineering and computer sciences from the University of California, Berkeley, in 1998.

$\mathrm{He}$ is currently an Associate Professor in the Department of Electrical and Systems Engineering, and the Director of the GRASP Laboratory, the University of Pennsylvania, Philadelphia. He also holds secondary appointments in the Departments of Computer and Information Sciences, and Mechanical Engineering and Applied Mechanics. He has published over one hundred articles in the areas of hybrid systems, hierarchical control systems, distributed control systems, nonlinear control systems, and geometric control theory, with applications to robotics, unmanned aerial vehicles, and biomolecular networks. He coedited Hybrid Systems: Computation and Control (New York: Springer-Verlag, 2004).

Dr. Pappas is the recipient of a National Science Foundation (NSF) Career Award in 2002, as well as the 2002 NSF Presidential Early Career Award for Scientists and Engineers (PECASE). He received the 1999 Eliahu Jury Award for Excellence in Systems Research from the Department of Electrical Engineering and Computer Sciences, University of California, Berkeley. His and his students' papers were finalists for the Best Student Paper Award at the IEEE Conference on Decision and Control $(1998,2001,2004)$ and the American Control Conference $(2001,2004)$. He is currently serving as an Associate Editor for the IEEE TRANSACTIONS ON AUTOMATIC CONTROL. 